\title{
Tracing the magnetic field and other properties of G351.417+0.645 at subarcsecond scales with the Long Baseline Array
}

\author{
T. Chanapote ${ }^{\odot},{ }^{1,2,3 \star}$ K. Asanok, ${ }^{1,3 \star}$ R. Dodson ${ }^{\odot},{ }^{2}$ M. Rioja ${ }^{\odot}, 2,4,5$ J. A. Green ${ }^{6}$ and \\ B. Hutawarakorn Kramer 3,7 \\ ${ }^{1}$ Department of Physics, Faculty of Science, Khon Kaen University, 123 Moo 16, T. Nai-Muang, A. Muang, Khon Kaen 40002, Thailand \\ ${ }^{2}$ International Centre for Radio Astronomy Research, The University of Western Australia, M468, 35 Stirling Highway, Crawley, Perth, WA 6009, Australia \\ ${ }^{3}$ National Astronomical Research Institute of Thailand, 260 Moo 4, T. Donkaew, A. Maerim, Chiangmai 50180, Thailand \\ ${ }^{4}$ CSIRO Astronomy and Space Science, 26 Dick Perry Avenue, Kensington WA 6151, Australia \\ ${ }^{5}$ Observatorio Astronómico Nacional (IGN), Alfonso XII, 3 y 5, E-28014 Madrid, Spain \\ ${ }^{6}$ CSIRO Astronomy and Space Science, Australia Telescope National Facility, PO Box 76, Epping, NSW 1710, Australia \\ ${ }^{7}$ Max-Planck-Institut für Radioastronomie, Auf dem Hügel 69, D-53121 Bonn, Germany
}

Accepted 2018 October 11. Received 2018 October 11; in original form 2018 April 17

\begin{abstract}
We present the results from the Australian Long Baseline Array (LBA) observations of the ground- and excited-state $\mathrm{OH}$ masers (1.7- and 6.0-GHz lines) at high spatial and velocity resolution towards the high-mass star-forming region G351.417+0.645. We obtained accurate spatial gradient magnetic fields from 21 and 23 Zeeman pairs at ground and excited states, respectively, providing magnetic field measurements between -6.4 and $+4.4 \mathrm{mG}$. In addition, we verify the reliability of magnetic field strengths measured from previous lower resolution observations. We found that $\mathrm{OH}$ maser features at $1665-$ and $6035-\mathrm{MHz}$ reveal the same trend of a reversal of magnetic field which we attribute, based on recent infrared observations to cloud fragmentation. In comparison with previous LBA observations 11 yr prior, we identify several matched Zeeman pairs. We found no significant changes of magnetic field strengths, directions, or radial velocities from the matched Zeeman pairs between these two epochs (although there were small internal proper motions). This implies quite stable physical conditions in this region. Moreover, we also analysed the physical conditions from the coincidence of $\mathrm{OH}$ maser features from different transitions based on current $\mathrm{OH}$ maser models.
\end{abstract}

Key words: masers-polarization-methods: data analysis-astrometry-ISM: kinematics and dynamics - ISM: magnetic fields.

\section{INTRODUCTION}

The study of the early stages of high-mass $\left(\geq 8 \mathrm{M}_{\odot}\right)$ stars is limited by their dense and thick environments (opaque to visible light) and scarcity (short lifetime). However, the increasing development of the instruments for multiwavelength (i.e. infrared (IR), millimetre (mm), submillimetre (submm), radio) observations allows for better understanding of this field (Beuther et al. 2000; Marseille et al. 2010; Sakai et al. 2010). At radio wavelengths, cosmic masers - naturally occurring beamed radiation from molecules in interstellar mediums - become a crucial tool to study high-mass starforming regions (HMSFRs), for example, tracing their evolutionary sequences (Ellingsen et al. 2007; Breen \& Ellingsen 2012). Various species of cosmic maser are often detected towards HMSFRs including hydroxyl $(\mathrm{OH})$, methanol $\left(\mathrm{CH}_{3} \mathrm{OH}\right)$, water $\left(\mathrm{H}_{2} \mathrm{O}\right)$ (Fish 2007;

^E-mail: t.chanapote@gmail.com (TC); kitiyanee@ narit.or.th (KA)
Bartkiewicz \& van Langevelde 2012); among them, $\mathrm{OH}$ are of several strong maser lines and easily detectable with large Zeeman splitting coefficients suitable for magnetic field study (Fish et al. 2003). There are many properties, we can study through maser observations, especially in the case of $\mathrm{OH}$, including (1) morphology (Slysh et al. 2002; Hutawarakorn \& Cohen 2005); (2) magnetic fields (Davies 1974; Fish \& Reid 2007; Caswell, Kramer \& Reynolds 2011); (3) kinematics (Argon, Reid \& Menten 2003; Trinidad et al. 2013); and (4) physical properties (Etoka, Cohen \& Gray 2005).

This paper reports the results from $\mathrm{OH}$ maser observations obtained in 2012 towards the well-known HMSFR G351.417+0.645. This source is located inside the cometary ultra compact H II (UCHII) region NGC 6334F - the smallest and youngest clump within the optically thin molecular cloud complex NGC 6334 (Rodriguez, Canto \& Moran 1982) one of the nearest HMSFRs to the Sun at a parallax measured distance of $1.34_{-0.12}^{+0.15} \mathrm{kpc}$ (Reid et al. 2014). Based on far-infrared (FIR) observation of McBreen et al. (1979), NGC 6334F is within the NGC 6334I cluster that is 
extremely complex and associated with multifrequency emissions with multiple emitting systems including near-infrared (NIR; Persi et al. 1996), mid-infrared (MIR; Persi et al. 1998; Kraemer \& Jackson 1999; De Buizer, Pina \& Telesco 2000; De Buizer et al. 2002), FIR (Harvey \& Gatley 1983), and mm (Hunter et al. 2006; Brogan et al. 2016). Besides, this region is also abundant in maser emission, and these have been continuously observed for a long period of time by single dishes, interferometric arrays and very long baseline interferometers (VLBI) including OH (Lo et al. 1975; Kent \& Mutel 1982; Gaume \& Mutel 1987; Zheng 1989; Caswell \& Vaile 1995; Argon, Reid \& Menten 2000; Caswell 2003; Caswell et al. 2011; Caswell, Green \& Phillips 2013; Hunter et al. 2018), $\mathrm{CH}_{3} \mathrm{OH}$ (Caswell 1997; Walsh et al. 1998; Brogan et al. 2016; Hunter et al. 2018), and $\mathrm{H}_{2} \mathrm{O}$ (Forster \& Caswell 1989; Migenes et al. 1999; Brogan et al. 2016). Within the UCHII region NGC 6334F, OH and $\mathrm{CH}_{3} \mathrm{OH}$ masers are distributed along the north-western boundary while $\mathrm{H}_{2} \mathrm{O}$ masers were only detected in the north.

Caswell et al. (2011) performed the first VLBI high spatial and velocity resolution observations $\left(\sim 0.05 \operatorname{arcsec}\right.$ and $\sim 0.1 \mathrm{~km} \mathrm{~s}^{-1}$, respectively) of excited state $\mathrm{OH}$ masers $(6030$ and $6035 \mathrm{MHz})$ inside NGC 6334F using the Australian Long Baseline Array (LBA). They provided the first well-characterized spatial gradient magnetic field measurements in this region.

In the current study, we present follow-up $\mathrm{OH}$ maser observations of this region in both ground and excited states using the LBA with compatible high spatial and velocity resolutions. There are three main purposes for this work: (1) to obtain the first spatial gradient magnetic field measurements from both ground (1665, 1667, and $1720 \mathrm{MHz}$ ) and excited (6030 and $6035 \mathrm{MHz}$ ) transitions and compare with previous comparable and lower resolution observations; (2) to study magnetic field variations and kinematics over an 11-yr time span through the comparison between comparable LBA high resolution observations; and (3) to study the physical conditions in this region by comparing our observational results with current $\mathrm{OH}$ maser models.

In Sections 2 and 3 of this paper, we describe our observations, data reduction process, and data analysis. In Section 4, we present the results and discussions of our observations together with the comparisons with previous observations and models. In Section 5, we summarize our findings from this study.

\section{OBSERVATIONS}

Experiments V452A and V452B were observed using the Australian VLBI antennas including the Australia Telescope Compact Array (ATCA), Ceduna (Cd), Hobart (Ho), Mopra (Mp), and Parkes (Pa) as part of the LBA as summarized in Table 1. The experiment consisted of observations at two bands, on the 8th and 10th of 2012 August, covering the 6030- and 6035-MHz lines together with 6.7$\mathrm{GHz} \mathrm{CH}_{3} \mathrm{OH}$ line and 1665-, 1667-, and 1720-MHz lines, respectively. ATCA, Mp, and $\mathrm{Pa}$ are run by CSIRO and all are in New South Wales on the east coast of Australia. Ho and $\mathrm{Cd}$ are run by the University of Tasmania and are in Tasmania and South Australia, respectively. The baseline lengths range from $\sim 100 \mathrm{~km}$ (ATCA to $\mathrm{Mp}$ ) to $\sim 1400 \mathrm{~km}$ (ATCA to Ho) and $\sim 1500 \mathrm{~km}$ (ATCA to Cd) for 1.7 and $6.0 \mathrm{GHz}$, respectively, providing good sensitivity to both compact and more extended emission. Note that, however, Cd only observed the excited-state $\mathrm{OH}$ main lines (i.e. 6030 and $6035 \mathrm{MHz}$ ) and $\mathrm{Ho}$ only observed the $\mathrm{CH}_{3} \mathrm{OH}$ line (i.e. $6668 \mathrm{MHz}$ ) while, at $1.7 \mathrm{GHz}, \mathrm{Cd}$ did not observe and Ho only observed the groundstate $\mathrm{OH}$ main lines (i.e. 1665 and $1667 \mathrm{MHz}$ ). The observing set-up comprised four $16-\mathrm{MHz}$ (wide-band) intermediate frequen-
Table 1. The experiments V452A and V452B.

\begin{tabular}{|c|c|c|}
\hline Projects & V452A & V452B \\
\hline Observing dates & 2012 August 8 & 2012 August 10 \\
\hline \multirow[t]{4}{*}{ Target sources } & G351.417+0.645 & G351.417+0.645 \\
\hline & G351.445+0.660 & G351.445+0.660 \\
\hline & G351.581-0.353 & G351.581-0.353 \\
\hline & G351.775-0.536 & G351.775-0.536 \\
\hline \multirow[t]{5}{*}{ Calibrators } & $1718-649$ & $1718-649$ \\
\hline & $1729-373$ & $1729-373$ \\
\hline & J1640-3727 & $\mathrm{J} 1640-3727$ \\
\hline & $1921-293$ & $1921-293$ \\
\hline & $1934-638$ & $1934-638$ \\
\hline \multirow[t]{2}{*}{ Antennas } & ATCA, Cd, Ho & ATCA, Ho, Mp \\
\hline & $\mathrm{Mp}, \mathrm{Pa}$ & $\mathrm{Pa}$ \\
\hline Shortest baseline & $113 \mathrm{~km}$ & $113 \mathrm{~km}$ \\
\hline Longest baseline & $1504 \mathrm{~km}$ & $1396 \mathrm{~km}$ \\
\hline \multirow[t]{3}{*}{ Transitions (MHz) } & 6030 & 1665 \\
\hline & 6035 & 1667 \\
\hline & 6668 & 1720 \\
\hline \multicolumn{3}{|l|}{ Bandwidth (MHz) } \\
\hline -Wide band & 16 & 16 \\
\hline -Narrow band & 4 & 2 \\
\hline \multicolumn{3}{|l|}{ Subbands (IFs) } \\
\hline -Wide band & 4 & 4 \\
\hline -Narrow band & 3 & 3 \\
\hline \multicolumn{3}{|l|}{ Spectral channels } \\
\hline -Wide band & 32 & 32 \\
\hline -Narrow band & 2048 & 4096 \\
\hline Velocity resolution & $0.10 \mathrm{~km} \mathrm{~s}^{-1}$ & $0.09 \mathrm{~km} \mathrm{~s}^{-1}$ \\
\hline
\end{tabular}

cies (IFs) and three narrow-band IFs with bandwidths of 4- and 2-MHz for 6.0/6.7- and 1.7-GHz lines, respectively. The IFs were spread to cover the maser lines at 6030,6035 , and $6668 \mathrm{MHz}$ and 1665,1667 , and $1720 \mathrm{MHz}$, respectively. The observed data in nominal Left- and Right-Hand Circular Polarizations (LHCP and RHCP) were recorded to the LBA disc-based recorder system. For the observation, 10-min scans on fringe finding sources 1934-638 and 1921-293 were observed for bandpass and clock offset calibrations, along with 2-min scans on delay calibrator sources 1729-373 and 1718-259. The four well-known $\mathrm{OH}$ masers sources were observed: G351.417+0.645, G351.445+0.660 (observed communally with the previous source), G351.581-0.353 and G351.775-0.536. This paper reports only on the G351.417+0.645 observations at the pointing centre $\mathrm{RA}=17^{\mathrm{h}} 20^{\mathrm{m}} 53.37$ and Dec. $=-35^{\circ} 47^{\prime} 1^{\prime \prime} .2$ (J2000), while the results of the other target sources together with the methanol line will be separately published later. Compact strong polarization, phase-referencing, and amplitude calibrators were also observed with each maser source, with a cycle time of about 30 minutes. The data were correlated using DiFX. The narrow-band IFs were split into 4096 and 2048 channels, providing channel separations of 0.488 and $1.953 \mathrm{kHz}$ or velocity resolutions of 0.09 and 0.1 $\mathrm{km} \mathrm{s}^{-1}$ at 1.7 and $6.0 \mathrm{GHz}$, respectively.

\section{DATA REDUCTION AND ANALYSIS}

The calibration process was mainly performed using the AIPS (Astronomical Image Processing System) reduction package (Greisen 2003). We have followed the standard data reduction process for spectral line (Caswell 1997; Caswell et al. 2011) for both 1.7 and 6.0 GHz including (1) flagging of data, (2) instrument and environment corrections, (3) phase and amplitude corrections, (4) realignment of the channels, (5) phase referencing to the calibra- 
tor, and then to the brightest features. The first three steps were performed with continuum data. The source 1921-293 was used as phase tracking and amplitude calibrator. All solutions were applied to the spectral line data before proceeding to the last two steps. For the spectral line data, the systemic velocity was set to $-10 \mathrm{~km} \mathrm{~s}^{-1}$ at rest frequencies for all five $\mathrm{OH}$ lines: 6030.7470 , 6035.0920, 1665.4018, 1667.3590, and 1720.5300 MHz. For the final step, firstly the brightest features at 1665 and $6035 \mathrm{MHz}$ were phase referenced to the nearest calibrator $1729-373$ in which we obtain the interband astrometric errors (see Section A2). Finally, the Stokes LL high-quality images of the brightest channels at 1665 and $6035 \mathrm{MHz}$, created using DIFMAP (an interactive editing and mapping software) (Shepherd 1997), were used to self-calibrate for 1665- and 6035-MHz lines and phase reference to 1665-, 1667-, and $6030-\mathrm{MHz}$ lines; this provides the intraband astrometric errors (see Section A3).

All procedures and criteria for data analysis are defined as follows:

Image Cubes: After finishing data calibration, image cubes were created using IMAGR task in AIPS with image size $4096 \times 4096$ pixels, cell size 0.002 arcsec $\times 0.002$ arcsec for all transitions consisting of 100 and 150 channels for 1.7 and $6.0 \mathrm{GHz}$ covering their ranges of radial local standard of rest (LSR) velocity $\left(V_{\mathrm{LSR}}\right),{ }^{1}$ approximately from -15 to -6 and -17 to $-3 \mathrm{~km} \mathrm{~s}^{-1}$, respectively. Note that for $6.0 \mathrm{GHz}$, we excluded the $\mathrm{Cd}$ antenna in imaging (due to the low sensitivity from $\mathrm{Cd}$ long baselines) to improve the SNR. The restoring beamwidths at $1665 / 1667$ and $6030 / 6035 \mathrm{MHz}$ are $\sim 0.03 \operatorname{arcsec} \times 0.013 \operatorname{arcsec}$ and $\sim 0.05 \operatorname{arcsec}$ $\times 0.015$ arcsec with position angles of $\sim 88^{\circ}$ and $75^{\circ}$, respectively, while at $1720 \mathrm{MHz}$, lacking of Ho antenna, the restoring beamwidth is $\sim 0.13 \operatorname{arcsec} \times 0.05 \operatorname{arcsec}$ with position angle of $\sim-78^{\circ}$. In all considered channels, the image rms noise varies from 0.01 to $0.80 \mathrm{Jy}$ depending on the peak intensities; the average values at 1665/1667 and $1720 / 6030 / 6035 \mathrm{MHz}$ are $\sim 0.1$ and $0.03 \mathrm{Jy}$, respectively.

Maser feature: We used CASA viewer (in CASA package) to search and perform 2D Gaussian fitting to all emission peaks across images. Maser features were defined based on two main following criteria: (1) their emissions are detected at least in three successive frequency channels with minimum $\mathrm{SNR}^{2}$ of three and (2) the peak position offsets are within the restoring beamwidths. Then we calculated intensity-weighted position and velocity together with the positional standard deviations $(\sigma)$ for each feature from its component spots. The average $\sigma$ for 1665-, 1667-, 6030-, and 6035-MHz images is $\sim 2$ mas, while for $1720-\mathrm{MHz}$ images the value is increased to $\sim 5$ mas due to its wider restoring beamwidth.

Coincidence of maser features: Maser features from different transitions are defined to be coincident if they lie within their absolute positional uncertainty which is the quadrature sum of all positional errors including thermal noise, interband and intraband (only for 1667-, 1720-, and 6030-MHz images) astrometric errors

\footnotetext{
${ }^{1}$ The velocity with respect to the LSR which is the perfectly circular orbit around the galactic centre at the sun distance (Schönrich, Binney \& Dehnen 2010). The $V_{\text {LSR }}$ is derived from Doppler shift of the observed spectral frequency with respect to the rest frequency:
}

$V_{\mathrm{LSR}}=V_{c}-\left(\frac{f_{s}-f_{r}}{f_{r}}\right) \times c$,

where $V_{c}$ is the central reference LSR velocity, $f_{s}$ and $f_{r}$ are the observed source frequency and rest frequency, respectively, and $c$ is the speed of light. ${ }^{2}$ Signal-to-noise ratio, the ratio between peak flux density and rms noise. (see the Appendix) and $\sigma$ written as following equations with average values (in mas unit):

$$
\begin{aligned}
& 1665 \mathrm{MHz}: \sqrt{1.6^{2}+11.4^{2}+\sigma^{2}} \approx 12, \\
& 1667 \mathrm{MHz}: \sqrt{1.6^{2}+11.4^{2}+0.5^{2}+\sigma^{2}} \approx 12, \\
& 1720 \mathrm{MHz}: \sqrt{1.6^{2}+11.4^{2}+13.2^{2}+\sigma^{2}} \approx 19, \\
& 6030 \mathrm{MHz}: \sqrt{1.0^{2}+1.3^{2}+0.3^{2}+\sigma^{2}} \approx 3, \\
& 6035 \mathrm{MHz}: \sqrt{1.0^{2}+1.3^{2}+\sigma^{2}} \approx 3 .
\end{aligned}
$$

Zeeman pairs: The Zeeman pairs are established from LHCP and RHCP maser features of the same transitions having position offsets within their absolute positional uncertainties. The magnetic field strengths are calculated from following equation:

$B=\left(V_{\mathrm{RR}}-V_{\mathrm{LL}}\right) / C_{Z}$,

where $V_{\mathrm{RR}}$ and $V_{\mathrm{LL}}$ are the velocities of RHCP and LHCP maser features, respectively, and $\mathrm{C}_{Z}$ is the Zeeman splitting coefficient. The Zeeman splitting coefficients used in this work were adopted from Green et al. (2012), Caswell et al. (2011), Caswell (2004), and Caswell (2003) that are 0.590, 0.354, 0.113, 0.079, $0.0564 \mathrm{~km} \mathrm{~s}^{-1}$ $\mathrm{mG}^{-1}$ for $1665,1667,1720,6030$, and $6035 \mathrm{MHz}$, respectively. The negative and positive signs of magnetic field strength from this equation imply the magnetic direction directed towards and away from us, respectively. We have adopted the IEEE definition for RHCP and LHCP and the IAU convention for Stokes V, i.e. RHCP would be seen by an observer as counterclockwise circular polarization.

Radial velocity: We considered only the maser features having their matched Zeeman pairs and averaged the velocities of RHCP and LHCP features from each Zeeman pair to estimate the demagnetized velocity which we determined to represent the velocity of the maser emission if there was no magnetic field present.

Proper motion: Proper motion is defined when the position difference of the same maser features from different epochs are larger than their positional uncertainties. In this work, we compared the same $\mathrm{OH}$ maser features in the 6.0-GHz lines with Caswell et al. (2011). The brightest LHCP feature at $6035 \mathrm{GHz}$ of two epochs was used as a reference feature, as a result, this is the internal proper motion with respect to the reference feature. Then, the positional uncertainties includes only intraband astrometric errors of 0.3 mas when applying from $6035 \mathrm{MHz} \rightarrow 6030 \mathrm{MHz}$ and $\sigma$ as following equations with average values (in mas unit),

$6030 \mathrm{MHz}: \sqrt{0.3^{2}+\sigma^{2}} \approx 3$,

$6035 \mathrm{MHz}: \sigma \approx 2$.

The positional uncertainties of maser features from Caswell et al. (2011) are assumed to be equivalent to those from this work. The criteria for defining the same $\mathrm{OH}$ features between the two epochs are location proximity, trend of spatial distribution with respect to the reference feature and relevance of their radial velocities. Linear displacement $(\vec{S})$ that each $\mathrm{OH}$ maser feature moves in 11-yr time span is calculated by adopting the target source distance $(D)$ of $1.34_{-0.12}^{+0.15} \mathrm{kpc}$ from Reid et al. (2014) as follow:

$\vec{S}=\vec{\theta} D$,

where $\vec{\theta}$ is the angular displacement. The transverse velocities $\left(\vec{V}_{T}\right)$ along the sky plane are calculated from the linear displacement $(S)$ divided by length of time $(t)$ between the two epochs:

$\vec{V}_{T}=\vec{S} / t$. 


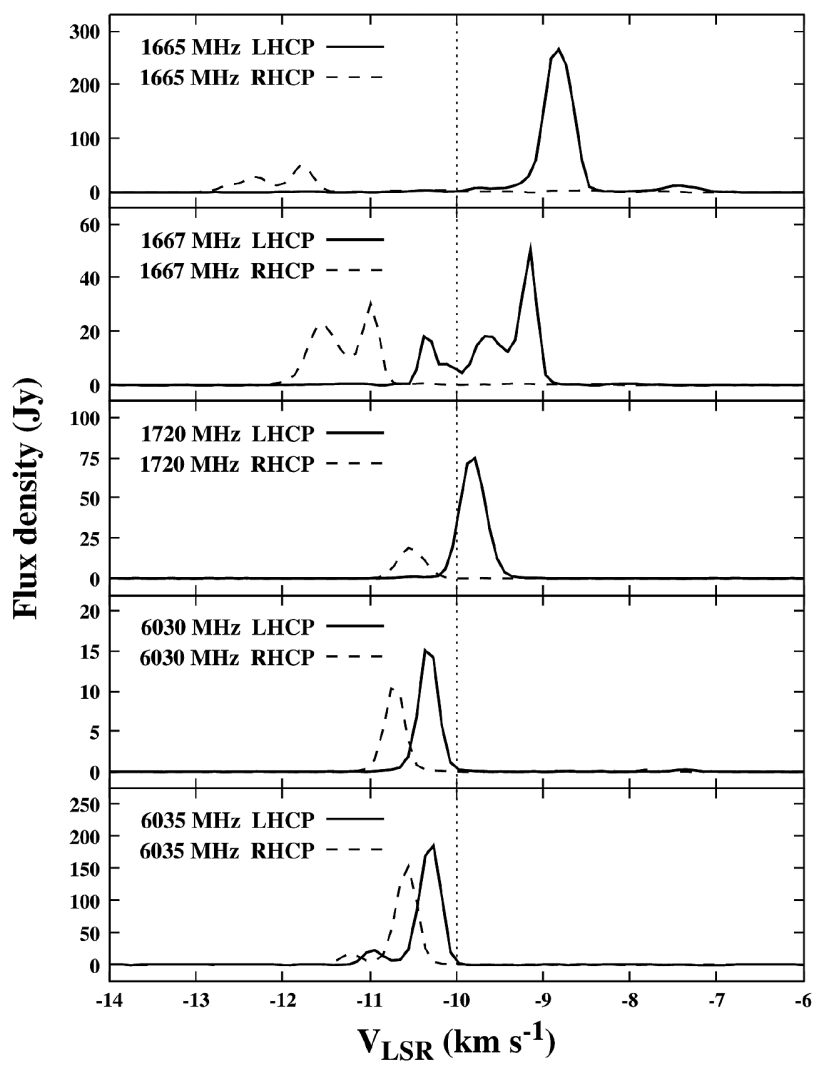

Figure 1. The vector averaged spectra of the ground and excited state transitions with respect to LSR velocities from the shortest baseline with the highest sensitivity, i.e. ATCA and Mp. The systemic velocity is set to $-10 \mathrm{~km} \mathrm{~s}^{-1}$ at the rest frequencies of all transitions shown as the vertical dot line.

\section{RESULTS AND DISCUSSION}

\subsection{OH spectra}

The vector averaged spectra from the ground and excited state transitions with respect to LSR velocities are shown in Fig. 1. The correlated spectra are chosen from the shortest baseline (i.e. ATCA and $\mathrm{Mp}$ ) to obtain the highest sensitivity. $\mathrm{OH}$ maser spectra from all transitions show the same trend of higher flux density in the LHCP than in the RHCP. The strongest lines in the ground- and excitedstate transitions are 1665 and $6035 \mathrm{MHz}$ that have flux densities in the order of a few hundred Jy.

The $\mathrm{OH}$ maser features were searched within the velocity range between -14 and $-6 \mathrm{~km} \mathrm{~s}^{-1}$. The properties of this region analysed from those maser features are divided into four sections as follows: Section 4.2 - Morphology, Section 4.3 - Magnetic fields, Section 4.4 - Kinematics, and Section 4.5 - Physical properties.

\subsection{Morphology}

The $\mathrm{OH}$ maser distributions at all observed transitions from both LHCP and RHCP are shown in Fig. 2. The reference position is taken to be the location of the $6035-\mathrm{MHz}$ brightest LHCP feature at $\mathrm{RA}(\mathrm{J} 2000)=17^{\mathrm{h}} 20^{\mathrm{m}} 53^{\mathrm{s}} .3716$ and Dec. $(\mathrm{J} 2000)=-35^{\circ} 47^{\prime} 1^{\prime \prime} .608$ to which all $\mathrm{OH}$ maser features at $6.0 \mathrm{GHz}$ are phase-referenced. The 1665-MHz brightest LHCP feature position is at RA (J2000) $=17^{\mathrm{h}} 20^{\mathrm{m}} 53^{\mathrm{s}} .3713$ and Dec. $(\mathrm{J} 2000)=-35^{\circ} 47^{\prime} 1^{\prime \prime} .612$ approximately 0.006 arcsec offset from the $6035-\mathrm{MHz}$ reference feature and similarly all $\mathrm{OH}$ maser features at $1.7 \mathrm{GHz}$ are phase-referenced to this position. Additionally, the brightest LHCP features from both 1.7 and $6.0 \mathrm{GHz}$ are phase-referenced to the common external quasar 1729-373. Based on this process, all OH maser features are well astrometrically registered as described in the Appendix. There are $142 \mathrm{OH}$ maser features detected from all five transitions as listed in Tables 2-5. We found 56, 24, 10, 10, and 42 features at 1665 (black), 1667 (green), 1720 (magenta), 6030 (red), and 6035 (blue) MHz, respectively, including both LHCP and RHCP features. All $\mathrm{OH}$ maser features in this source are distributed over an area of $\sim 2.0 \operatorname{arcsec} \times 3.5 \operatorname{arcsec}\left(\right.$ or $2680^{3}$ au $\times 4690^{3} \mathrm{au}$ ). The distribution of all $\mathrm{OH}$ masers from both 1.7 and $6.0 \mathrm{GHz}$ transitions trace an arc-shape along the north-west boundary of the UCHII region NGC 6334F observed with the VLA at 5-cm wavelength and the 3-mm ALMA dust continuum clump MM3 (red dash and black solid contours in Fig. 2, respectively) (Brogan et al. 2016).

We compared our $\mathrm{OH}$ maser features in the ground-state transitions with previous lower spatial and velocity resolution observations of Argon et al. (2000) and Gaume \& Mutel (1987) obtained with the VLA. Argon et al. (2000) found 17, 14, and 3 features at 1665,1667 , and $1720 \mathrm{MHz}$, respectively, as listed in their table 85, while Gaume \& Mutel (1987) detected 14, 8, and 4 features at 1665 , 1667 , and $1720 \mathrm{MHz}$, respectively, as listed in their table 1. Our considerably larger number of $\mathrm{OH}$ maser features probably results from the finer spatial and velocity resolution of our observations, which resolve some blended features from previous ground-state observations at lower resolution. For the positional comparisons, due to their large absolute position uncertainties $(\sim 0.3 \mathrm{arcsec})$, we set the $1665-\mathrm{MHz}$ brightest LHCP features from our work and their works to the same position. However, there are uncertainties to be considered as following. Using the VLA, Argon et al. (2000) reported Gaussian fitting errors of 0.01 arcsec, while those of Gaume \& Mutel (1987)'s work are much higher $\sim 0.15$ arcsec. Our Gaussian fitting errors are much smaller (in micro-arcsecond scale), and $\sigma_{R A}$ and $\sigma_{D e c}$ are in mas scale (generally less than 10 mas). From the comparison plot (not shown), we found the same trend of maser distributions at 1665 and $1667 \mathrm{MHz}$ along the north-west boundary of the UCHII region NGC $6443 \mathrm{~F}$ where there is a sharp increase in flux density as shown in figs 35 and 24 of Argon et al. (2000) and Gaume \& Mutel (1987), respectively. At $1720 \mathrm{MHz}$, there are obvious position shifts of $\mathrm{OH}$ maser features between our results and Argon et al. (2000)'s because the positions of $\mathrm{OH}$ maser features do not overlap within each other's errors. ${ }^{4}$ However, some of our 1720-MHz OH maser features below the reference centre are coincident with those of Gaume \& Mutel (1987) and we detected new $1720-\mathrm{MHz}$ features above the reference centre.

In the excited-state transitions, in comparison with recent VLA observations (epoch 2016) from Hunter et al. (2018) inside NGC $6334 \mathrm{~F}$ with comparable velocity resolution of $\sim 0.1 \mathrm{~km} \mathrm{~s}^{-1}$ and lower angular resolution of $0.79 \operatorname{arcsec} \times 0.25 \mathrm{arcsec}$, we found that their seven groups of $6.0-\mathrm{GHz} \mathrm{OH}$ masers $(\mathrm{OH} 1-\mathrm{OH} 7)$ as shown in their fig. 5 reproduce the features detected in our epoch

\footnotetext{
${ }^{3}$ This is calculated from the NGC 6334 distance of $\sim 1.34 \mathrm{kpc}$ from maser parallax measurements of Reid et al. (2014).

${ }^{4}$ Our approximate uncertainty of the relative positions of $1720 \mathrm{MHz}$ transitions to the brightest features at $1665 \mathrm{MHz}$ is approximately 13.2 mas as described in Section A3. Argon et al. (2000) suggested the uncertainties in relative position between different transitions should be from 1/4 to $1 / 2$ of the absolute position errors providing the approximate errors of $0.15 \mathrm{arcsec}$ and 0.50 arcsec in RA and Dec., respectively, while the total errors of Gaume \& Mutel (1987) are $\sim 0.3$ arcsec.
} 

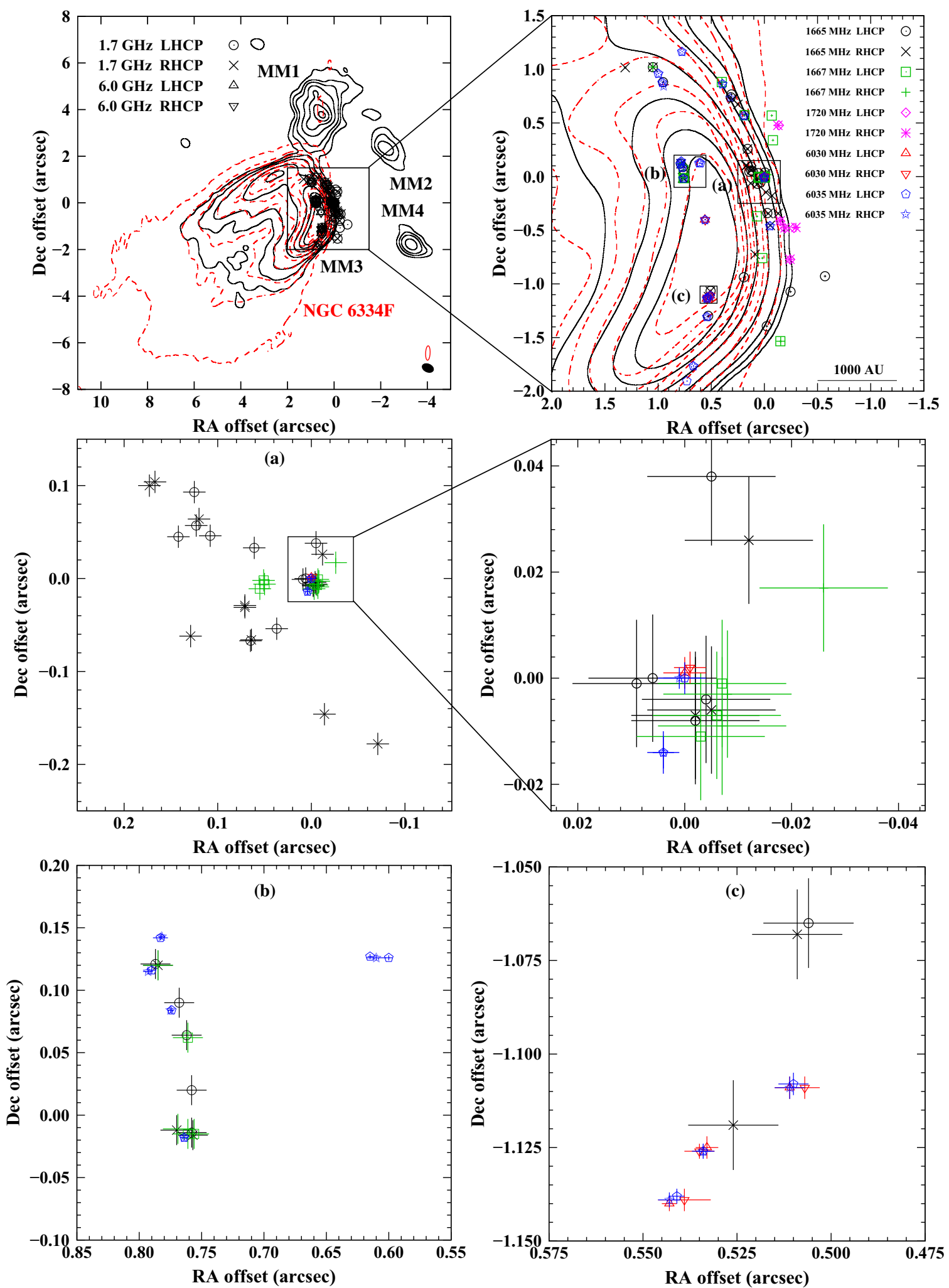

Figure 2. The top left image shows the $\mathrm{OH}$ masers at 1.7 (circle and cross represent LHCP and RHCP, respectively) and $6.0 \mathrm{GHz}$ (point-up and point-down triangles represent LHCP and RHCP, respectively) overlaid on the 5-cm UCHII NGC 6334F continuum [red dash contours: $4.2 \times 10^{-5} \mathrm{Jy}(4,50,100,200,300$, $400,500,600,700)$ ] and 3-mm dust continuum [black solid contours: $5 \times 10^{-5} \mathrm{Jy}(4,10,20,50,100,150,200)$ ] of the NGC 3664I multiclump system from Brogan et al. (2016) where the dust clump MM3 is coincident with the UCHII region NGC 6334F. The beamwidths of the continuum data with corresponding colours are displayed in the bottom-right corner. The top right image presents a magnification of the region where the masers are found where different symbols and colours represent different $\mathrm{OH}$ lines: $1665 \mathrm{MHz}$ (black circle and cross), $1667 \mathrm{MHz}$ (green square and plus), $1720 \mathrm{MHz}$ (magenta diamond and sparkle), $6030 \mathrm{MHz}$ (red up- and down-triangle), and $6035 \mathrm{MHz}$ (blue pentagon and star). The absolute positional uncertainties with the same colours as the symbols are also shown. The four lower panels present a magnification of three compact areas: (a), (b), and (c). 
Table 2. OH maser features from both LHCP and RHCP for G351.417+0.645 at $1665 \mathrm{MHz}$. Position offsets are relative to the brightest $6035 \mathrm{LHCP}$ feature at RA $(\mathrm{J} 2000)=17^{\mathrm{h}} 20^{\mathrm{m}} 53^{\mathrm{s}} .3716$ and Dec. $(\mathrm{J} 2000)=-35^{\circ} 47^{\prime} 1^{\prime \prime} .608$.

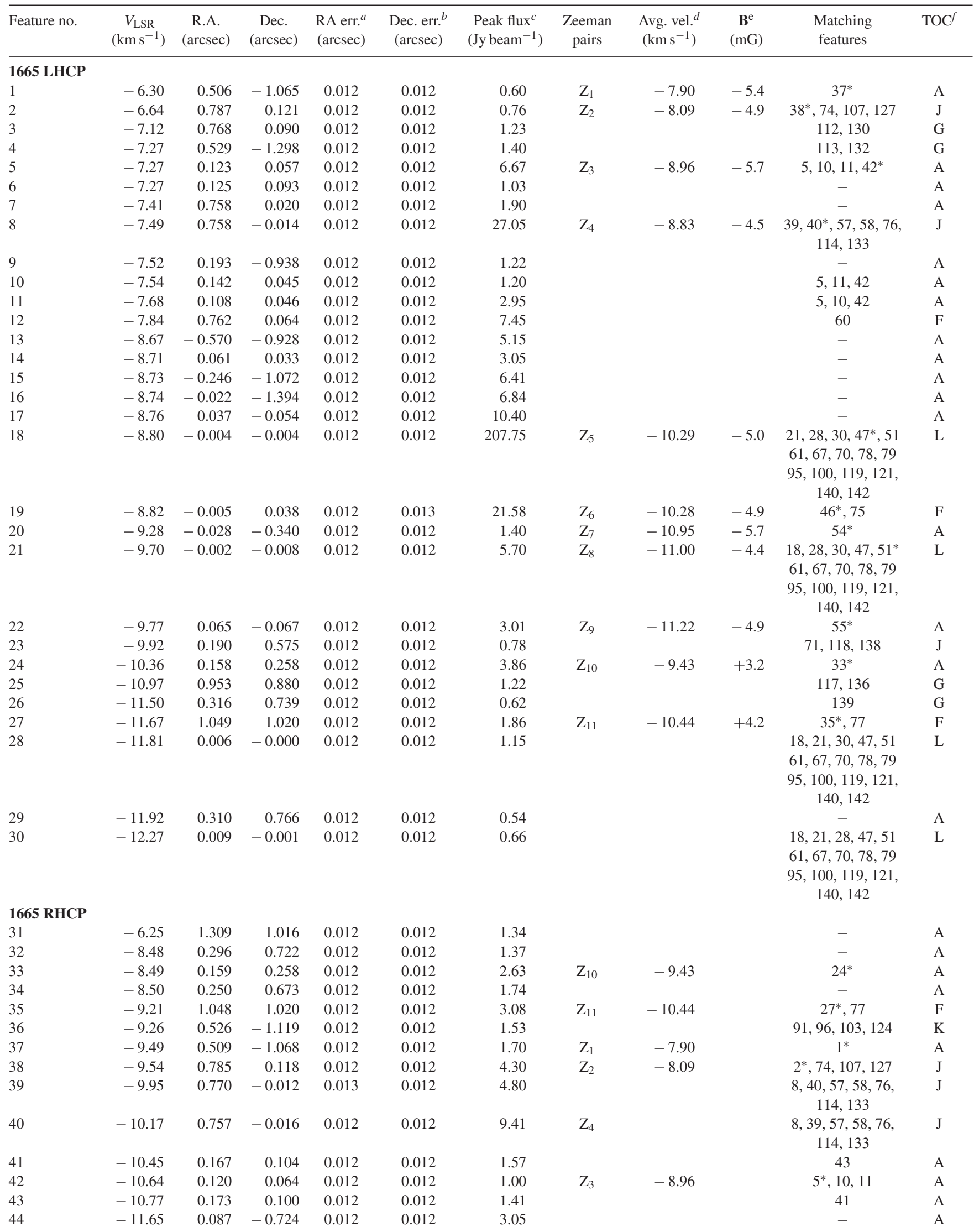


Table 2 - continued

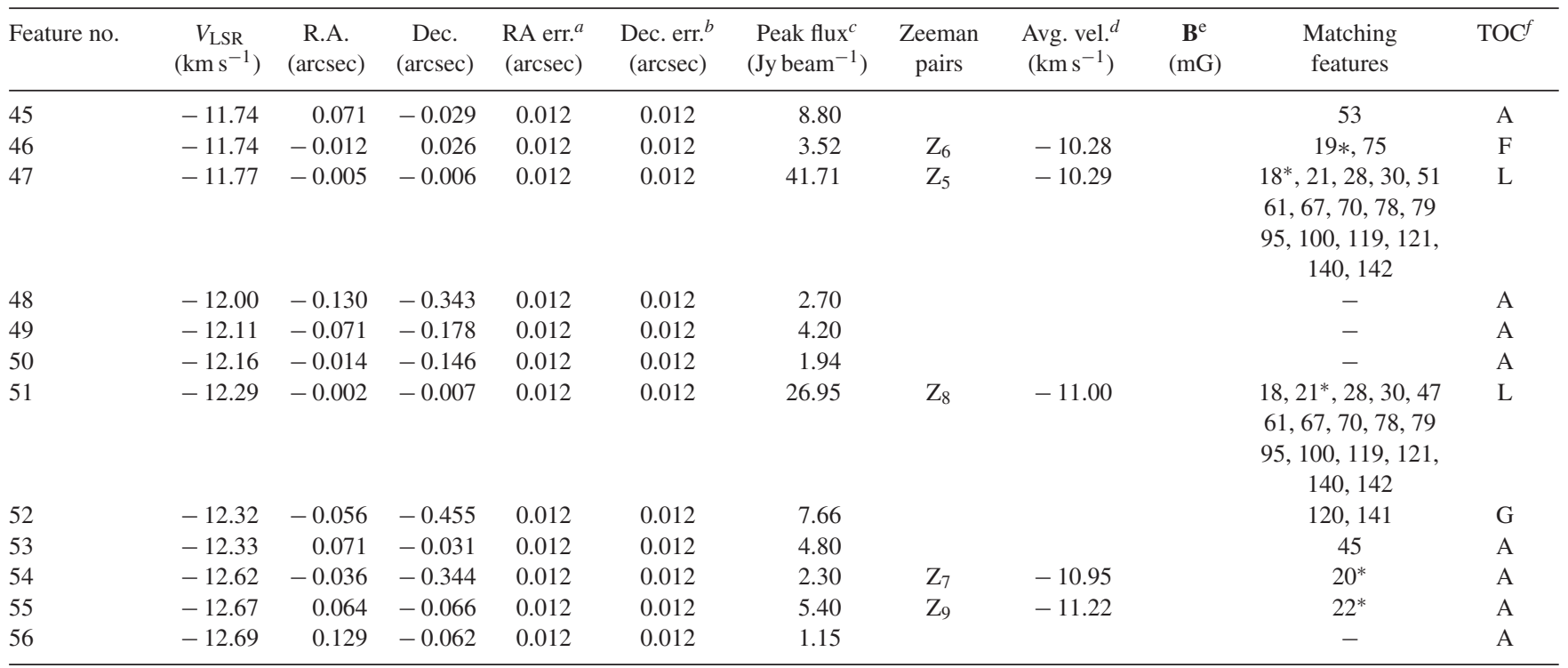

Notes. ${ }^{a, b}$ The absolute positional errors in RA and Dec offsets.

${ }^{c}$ The peak flux of the brightest component was taken to be the peak flux density of the maser feature. The average rms noise of $1665-\mathrm{MHz}$ images is $\sim 0.06 \mathrm{Jy}$ with maximum value of $0.7 \mathrm{Jy}(\sim 0.4$ per cent of the peak flux $)$ in the brightest channel.

${ }^{d}$ The average (demagnetized) velocity from the velocities of LHCP and RHCP features of each Zeeman pair. ${ }^{e}$ The strength of magnetic field (B) can be calculated from equation (3) and the maximum uncertainty of $\mathbf{B}$ measurement $(\Delta \mathbf{B})$ is $\pm 0.2 \mathrm{mG}$.

${ }^{f}$ Types of coincidence (TOC) including A (only $1665 \mathrm{MHz}$ ), B (only $1667 \mathrm{MHz}$ ), C (only $1720 \mathrm{MHz}$ ), D (only $6030 \mathrm{MHz}$ ), E (only $6035 \mathrm{MHz}$ ), F (1665 \& $1667 \mathrm{MHz}), \mathrm{G}(1665$ \& $6035 \mathrm{MHz}), \mathrm{H}(1667 \& 6035 \mathrm{MHz}), \mathrm{I}(6030$ \& $6035 \mathrm{MHz}), \mathrm{J}(1665$ \& 1667 \& $6035 \mathrm{MHz}), \mathrm{K}(1665$ \& 6030 \& $6035 \mathrm{MHz}) \mathrm{and} \mathrm{L}$ $(1665 \& 1667 \& 6030 \& 6035 \mathrm{MHz})$.

*The matched Zeeman pairs.

2012 observations distributed along the north-west boundary of the UCHII region NGC 6334F.

Moreover, we also compare the position of our $6.0-\mathrm{GHz} \mathrm{OH}$ maser features (epoch 2012) with those of Caswell et al. (2011) (epoch 2001; cf. their table 1) obtained with comparable high resolution observations. The phase reference feature of Caswell et al. (2011) is at RA (J2000) $=17^{\mathrm{h}} 20^{\mathrm{m}} 53.38$ and Dec. (J2000) $=-35^{\circ} 47^{\prime} 1^{\prime \prime}$.5. However, Caswell et al. (2011) reported a minimum absolute position uncertainty of $\sim 0.2$ arcsec $^{5}$ In order to make a comparison, we set the reference features of both epochs to be at the same position for more accurate comparison of the relative positions between two epochs. The overlay plot (not shown) of our 6.0-GHz maser features and those of Caswell et al. (2011) show almost exactly the same spatial distribution. 81 per cent (100 per cent matching features for the $6030 \mathrm{-MHz}$ transition and 76 per cent for the $6035 \mathrm{MHz}$ ) of all the features detected in this work in the 6.0GHz transitions were also detected by Caswell et al. (2011). The matching features are given in column 1 of Tables 4 and 5. At $6035 \mathrm{MHz}, 14 \mathrm{OH}$ maser features observed in the 2001 epoch were not detected by us; 13 of the 14 features had fluxes in the range 0.06-1.3 Jy and represented the weakest features in Caswell et al. (2011), while one of 14 features has a high flux of $3.7 \mathrm{Jy}$. Four of the 14 features were spectrally close to bright features and may have been confused. On the other hand, the other features are in high sensitivity channels, so their disappearance might be from flux variations due to some environmental changes over an 11-yr period. Moreover, at $6035 \mathrm{MHz}$, there are 10 new $\mathrm{OH}$ maser features de-

\footnotetext{
${ }^{5}$ This uncertainty was calculated by averaging derived values from different observing sessions.
}

tected in our observations, nine of which are weak (with fluxes in the range $0.2-0.7 \mathrm{Jy}$ ), and one of which (our feature 139) is a bright feature $(4.7 \mathrm{Jy})$. This is also probably the result of maser variation.

\subsection{Magnetic Fields}

The well-characterized spatial gradient magnetic fields measured from 21 and 23 clearly matched Zeeman pairs at 1.7 and $6.0 \mathrm{GHz}$, respectively, are shown in Fig. 3. We estimate the magnetic field strengths ranging from -6.4 to $+4.4 \mathrm{mG}$ as listed in Tables 2 5. Overall, the magnetic field directions are directed towards us with a reversal of magnetic field just below and above Galactic longitude 351.417; the assumption for the cause of the reversal will be explained in Section 4.3.1. The coincident Zeeman pairs from our results (located within each other's quadrature errors) tend to provide corresponding demagnetized velocities as listed in Table 6 that present the $\mathrm{OH}$ Zeeman pair associations, adding strength to their colocations.

In Group 1, there are two coincident Zeeman pairs, $\mathrm{Z}_{2}$ and $\mathrm{Z}_{32}$, from $1665-$ and $6035-\mathrm{MHz}$ transitions, respectively, with correspondent averaged (demagnetized) velocities and magnetic field strengths. In Group 2, we found three coincident Zeeman pairs, $\mathrm{Z}_{4}, \mathrm{Z}_{12}$, and $\mathrm{Z}_{38}$ from 1665-, 1667-, and 6035-MHz transitions, respectively. In Group 3, at the reference centre, we found several coincident Zeeman pairs from four transitions i.e. 1665, 1667, 6030, and $6035 \mathrm{MHz}$. Three Zeeman pairs, $\mathrm{Z}_{5}, \mathrm{Z}_{6}$, and $\mathrm{Z}_{8}$, are detected at $1665-\mathrm{MHz}$ transition. The $\mathrm{Z}_{6}$, a separated Zeeman pair in the north, is not coincident with the other Zeeman pairs; however, it is noteworthy that the demagnetized velocity and magnetic field strength of this Zeeman pair are in good agreement with those of $Z_{5}$. In addition, there are two Zeeman pairs from 1667-MHz transition, $\mathrm{Z}_{14}$ 
Table 3. OH maser features from both LHCP and RHCP for G351.417+0.645 at $1667 \mathrm{MHz}$. Position offsets are relative to the brightest $6035 \mathrm{LHCP}$ feature.

\begin{tabular}{|c|c|c|c|c|c|c|c|c|c|c|c|}
\hline Feature no. & $\begin{array}{c}V_{\mathrm{LSR}} \\
\left(\mathrm{km} \mathrm{s}^{-1}\right)\end{array}$ & $\begin{array}{c}\mathrm{RA} \\
(\operatorname{arcsec})\end{array}$ & $\begin{array}{c}\text { Dec. } \\
(\operatorname{arcsec})\end{array}$ & $\begin{array}{l}\text { RA err. }{ }^{a} \\
(\operatorname{arcsec})\end{array}$ & $\begin{array}{c}\text { Dec err. }{ }^{b} \\
(\operatorname{arcsec})\end{array}$ & $\begin{array}{c}\text { Peak flux }{ }^{c} \\
\left(\text { Jy beam }^{-1}\right)\end{array}$ & $\begin{array}{c}\text { Zeeman } \\
\text { pairs }\end{array}$ & $\begin{array}{l}\text { Avg. vel. }{ }^{d} \\
\left(\mathrm{~km} \mathrm{~s}^{-1}\right)\end{array}$ & $\begin{array}{c}\mathbf{B}^{e} \\
(\mathrm{mG})\end{array}$ & $\begin{array}{l}\text { Matching } \\
\text { features }\end{array}$ & $\mathrm{TOC}^{f}$ \\
\hline \multicolumn{12}{|l|}{1667 LHCP } \\
\hline 57 & -7.76 & 0.761 & -0.015 & 0.012 & 0.012 & 0.90 & $\mathrm{Z}_{12}$ & -8.57 & -4.6 & $\begin{array}{c}8,39,40,58 \\
76^{*} \\
114,133\end{array}$ & $\mathrm{~J}$ \\
\hline 58 & -8.14 & 0.756 & -0.015 & 0.012 & 0.012 & 3.07 & & & & $\begin{array}{c}8,39,40,57 \\
76 \\
114,133\end{array}$ & $\mathrm{~J}$ \\
\hline 60 & -8.45 & 0.761 & 0.062 & 0.012 & 0.012 & 1.81 & & & & 12 & F \\
\hline 61 & -9.19 & -0.007 & -0.001 & 0.012 & 0.012 & 24.56 & $\mathrm{Z}_{14}$ & -10.10 & -5.1 & $\begin{array}{c}18,21,28 \\
30,47 \\
51,67,70 \\
78^{*}, 79 \\
95,100,119 \\
121,140,142\end{array}$ & $\mathrm{~L}$ \\
\hline 65 & -9.55 & -0.069 & 0.569 & 0.012 & 0.012 & 3.07 & & & & - & B \\
\hline 66 & -9.60 & 0.050 & -0.006 & 0.012 & 0.012 & 3.43 & & & & 62,69 & B \\
\hline 67 & -9.62 & -0.006 & -0.007 & 0.012 & 0.012 & 14.40 & $\mathrm{Z}_{15}$ & -10.58 & -5.4 & $\begin{array}{c}18,21,28 \\
30,47 \\
51,61,70 \\
78,79^{*} \\
95,100,119 \\
121,140,142\end{array}$ & $\mathrm{~L}$ \\
\hline 68 & -9.69 & 0.070 & -0.370 & 0.012 & 0.012 & 3.22 & & & & - & B \\
\hline 69 & -10.29 & 0.055 & -0.011 & 0.012 & 0.012 & 2.50 & & & & 62,66 & B \\
\hline 70 & -10.30 & -0.003 & -0.011 & 0.012 & 0.012 & 15.48 & & & & $\begin{array}{c}18,21,28 \\
30,47 \\
51,61,67 \\
78,79 \\
95,100,119 \\
121,140,142\end{array}$ & $\mathrm{~L}$ \\
\hline \multicolumn{12}{|l|}{1667 RHCP } \\
\hline 74 & -8.89 & 0.785 & 0.120 & 0.012 & 0.012 & 1.93 & & & & $\begin{array}{c}2,38,107 \\
127\end{array}$ & $\mathrm{~J}$ \\
\hline 75 & -9.17 & -0.026 & 0.017 & 0.012 & 0.012 & 0.62 & & & & 19,46 & F \\
\hline 76 & -9.37 & 0.769 & -0.011 & 0.012 & 0.012 & 2.04 & $\mathrm{Z}_{12}$ & -8.57 & & $\begin{array}{l}8,39,40 \\
57^{*}, 58 \\
114,133\end{array}$ & $\mathrm{~J}$ \\
\hline 77 & -9.70 & 1.050 & 1.021 & 0.012 & 0.012 & 0.93 & & & & 27,35 & F \\
\hline 78 & -11.01 & -0.008 & -0.003 & 0.012 & 0.012 & 20.27 & $Z_{14}$ & -10.10 & & $\begin{array}{c}18,21,28 \\
30,47 \\
51,61^{*}, 67 \\
70,79 \\
95,100,119 \\
121,140,142\end{array}$ & $\mathrm{~L}$ \\
\hline 79 & -11.53 & -0.007 & -0.009 & 0.012 & 0.013 & 23.40 & $\mathrm{Z}_{15}$ & -10.58 & & $\begin{array}{c}18,21,28 \\
30,47 \\
51,61,67^{*} \\
70,78 \\
95,100,119 \\
121,140,142\end{array}$ & $\mathrm{~L}$ \\
\hline 80 & -12.30 & -0.153 & -1.534 & 0.013 & 0.012 & 0.90 & $\mathrm{Z}_{16}$ & -11.67 & & $72 *$ & B \\
\hline
\end{tabular}

Notes. ${ }^{a, b, d, f, * \text { See Table } 2 .}$

${ }^{c}$ The peak flux of the brightest component was taken to be the peak flux density of the maser feature. The average rms noise of $1667-\mathrm{MHz}$ images is $\sim 0.07 \mathrm{Jy}$ with maximum value of $0.5 \mathrm{Jy}$ ( $\sim 2$ per cent of the peak flux) in the brightest channel.

${ }^{e} \Delta \mathbf{B}$ is $\pm 0.3 \mathrm{mG}$. 
Table 4. OH maser features from both LHCP and RHCP for G351.417+0.645 at 1720 and $6030 \mathrm{MHz}$. Position offsets are relative to the brightest $6035 \mathrm{LHCP}$ feature.

\begin{tabular}{|c|c|c|c|c|c|c|c|c|c|c|c|}
\hline $\begin{array}{l}\text { Feature no. } \\
(\text { epoch 2001) } \dagger\end{array}$ & $\begin{array}{c}V_{\mathrm{LSR}} \\
\left(\mathrm{km} \mathrm{s}^{-1}\right)\end{array}$ & $\begin{array}{c}\mathrm{RA} \\
(\operatorname{arcsec})\end{array}$ & $\begin{array}{c}\text { Dec. } \\
(\operatorname{arcsec})\end{array}$ & $\begin{array}{l}\text { RA err. }^{a} \\
(\operatorname{arcsec})\end{array}$ & $\begin{array}{l}\text { Dec. err. }{ }^{b} \\
(\operatorname{arcsec})\end{array}$ & $\begin{array}{l}\text { Peak flux } \\
\left(\text { Jy beam }^{-1}\right)\end{array}$ & $\begin{array}{l}\text { Zeeman } \\
\text { pairs }\end{array}$ & $\begin{array}{l}\text { Avg. vel. } \\
\left(\mathrm{km} \mathrm{s}^{-1}\right)\end{array}$ & $\begin{array}{c}\mathbf{B}^{e} \\
(\mathrm{mG})\end{array}$ & $\begin{array}{l}\text { Matching } \\
\text { features }\end{array}$ & $\operatorname{TOC}^{f}$ \\
\hline \multicolumn{12}{|l|}{1720 LHCP } \\
\hline 81 & -9.79 & -0.278 & -0.479 & 0.020 & 0.018 & 13.70 & $Z_{17}$ & -10.15 & -6.4 & $88^{*}$ & $\mathrm{C}$ \\
\hline 82 & -9.79 & -0.193 & -0.482 & 0.018 & 0.018 & 16.20 & $\mathrm{Z}_{18}$ & -10.14 & -6.1 & $86^{*}$ & $\mathrm{C}$ \\
\hline 83 & -9.81 & -0.149 & -0.416 & 0.018 & 0.019 & 12.00 & $\mathrm{Z}_{19}$ & -10.15 & -6.0 & $87^{*}$ & $\mathrm{C}$ \\
\hline 84 & -9.81 & -0.137 & 0.478 & 0.019 & 0.019 & 14.20 & $\mathrm{Z}_{20}$ & -10.17 & -6.3 & $89^{*}$ & $\mathrm{C}$ \\
\hline 85 & -9.82 & -0.243 & -0.772 & 0.018 & 0.019 & 44.50 & $Z_{21}$ & -10.18 & -6.3 & $90^{*}$ & $\mathrm{C}$ \\
\hline \multicolumn{12}{|l|}{1720 RHCP } \\
\hline 86 & -10.48 & -0.194 & -0.477 & 0.018 & 0.019 & 3.40 & $\mathrm{Z}_{18}$ & -10.14 & & $82^{*}$ & $\mathrm{C}$ \\
\hline 87 & -10.49 & -0.149 & -0.411 & 0.021 & 0.020 & 2.20 & $\mathrm{Z}_{19}$ & -10.15 & & $83^{*}$ & $\mathrm{C}$ \\
\hline 88 & -10.51 & -0.296 & -0.473 & 0.019 & 0.018 & 2.90 & $\mathrm{Z}_{17}$ & -10.15 & & $81^{*}$ & $\mathrm{C}$ \\
\hline 89 & -10.51 & -0.127 & 0.478 & 0.018 & 0.019 & 4.50 & $\mathrm{Z}_{20}$ & -10.17 & & $84^{*}$ & $\mathrm{C}$ \\
\hline 90 & -10.53 & -0.240 & -0.770 & 0.018 & 0.020 & 12.30 & $\mathrm{Z}_{21}$ & -10.18 & & $85^{*}$ & $\mathrm{C}$ \\
\hline \multicolumn{12}{|l|}{6030 LHCP } \\
\hline $91(\mathrm{e})$ & -7.36 & 0.533 & -1.125 & 0.003 & 0.003 & 0.62 & $\mathrm{Z}_{22}$ & -7.57 & -5.3 & $36,96^{*}, 103,124$ & $\mathrm{~K}$ \\
\hline $92(d)$ & -7.89 & 0.543 & -1.140 & 0.002 & 0.002 & 0.20 & $\mathrm{Z}_{23}$ & -8.06 & -4.3 & $97^{*}, 106,126$ & I \\
\hline 93 (b) & -8.44 & 0.511 & -1.109 & 0.004 & 0.003 & 0.41 & $\mathrm{Z}_{24}$ & -8.64 & -5.1 & $99^{*}, 110,131$ & I \\
\hline $94(\mathrm{c})$ & -8.49 & 0.560 & -0.402 & 0.004 & 0.002 & 0.37 & $\mathrm{Z}_{25}$ & -8.63 & -3.4 & $98^{*}, 111,129$ & I \\
\hline 95 (a) & -10.32 & 0.000 & 0.001 & 0.004 & 0.003 & 11.92 & $\mathrm{Z}_{26}$ & -10.52 & -4.9 & $\begin{array}{c}18,21,28,30,47 \\
51,61,67,70,78 \\
79,100^{*}, 119,121 \\
140,142\end{array}$ & $\mathrm{~L}$ \\
\hline \multicolumn{12}{|l|}{6030 RHCP } \\
\hline $96(\mathrm{E})$ & -7.78 & 0.535 & -1.126 & 0.004 & 0.002 & 0.66 & $\mathrm{Z}_{22}$ & -7.57 & & $36,91^{*}, 103,124$ & K \\
\hline 97 (D) & -8.23 & 0.539 & -1.139 & 0.007 & 0.003 & 0.25 & $\mathrm{Z}_{23}$ & -8.06 & & $92^{*}, 106,126$ & I \\
\hline $98(\mathrm{C})$ & -8.76 & 0.557 & -0.402 & 0.003 & 0.002 & 0.38 & $\mathrm{Z}_{25}$ & -8.63 & & $94^{*}, 111,129$ & I \\
\hline 99 (B) & -8.84 & 0.507 & -1.109 & 0.004 & 0.003 & 0.36 & $\mathrm{Z}_{24}$ & -8.64 & & $93^{*}, 110,131$ & I \\
\hline $100(\mathrm{~A})$ & -10.71 & -0.001 & 0.002 & 0.003 & 0.003 & 8.59 & $\mathrm{Z}_{26}$ & -10.52 & & $\begin{array}{c}18,21,28,30,47 \\
51,61,67,70,78 \\
79,95^{*}, 119,121 \\
140,142\end{array}$ & $\mathrm{~L}$ \\
\hline
\end{tabular}

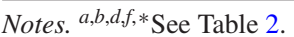

${ }^{c}$ The peak flux of the brightest component was taken to be the peak flux density of the maser feature. The average rms noise of $1720-\mathrm{MHz}$ images is $\sim 0.02$ Jy with maximum value of $0.8 \mathrm{Jy}(\sim 0.6$ per cent of the peak flux $)$ in the brightest channel, while for 6030 -MHz images, the average rms noise is $\sim 0.01 \mathrm{Jy}$ with maximum value of $0.07 \mathrm{Jy}(\sim 0.6$ per cent of the peak flux $)$ in the brightest channel.

${ }^{e}$ At 1720 and $6030 \mathrm{MHz}, \Delta \mathbf{B}$ are \pm 0.8 and $\pm 1.3 \mathrm{mG}$, respectively.

$\dagger$ The matching $\mathrm{OH}$ maser features detected by Caswell et al. (2011) are also given.

and $\mathrm{Z}_{15}$. Besides, there are one $6030-\mathrm{MHz}$ Zeeman pair, $\mathrm{Z}_{26}$, and two $6035-\mathrm{MHz}$ Zeeman pairs, $\mathrm{Z}_{43}$ and $\mathrm{Z}_{45}$. Note that $\mathrm{Z}_{45}$ has the bluest demagnetized velocity of $-11 \mathrm{~km} \mathrm{~s}^{-1}$ towards us implying that it is possibly from different location in the line of sight direction compared with the other Zeeman pairs in this group. In this case, higher spatial resolution observation is needed for verification.

Although Groups 1-3 show a mix of $1665 / 1667-\mathrm{MHz}$ and $6030 / 6035-\mathrm{MHz}$ coincidental maser emission, Groups 4-7 only exhibit 6030/6035-MHz coincidental maser emission.

Some of the coincident Zeeman pairs from the same transitions detected in this work might be blended in previous lower resolution observations. However, due to the correspondence of magnetic field values of the blended Zeeman pairs, the blending should not significantly affect the accuracy of previous magnetic field measurements from this transition.

At $6.0 \mathrm{GHz}$, we found that all five $6030-\mathrm{MHz}$ Zeeman pairs are coincident with $6035-\mathrm{MHz}$ Zeeman pairs including $\mathrm{Z}_{22}$ and $\mathrm{Z}_{29}$ in Group $4, Z_{23}$ and $Z_{31}$ in Group 5, $Z_{24}$ and $Z_{34}$ in Group 6, $Z_{25}$ and $Z_{35}$ in Group 7, and $Z_{26}$ and $Z_{43}$ in Group 3. The magnetic fields and demagnetized velocities from 6030- to 6035-MHz matching Zeeman pairs are in good agreement with each other proving the co-propagation of both transitions. Note that the matching Zeeman pairs $Z_{24}$ and $Z_{34}$ provide the largest difference of $0.8 \mathrm{mG}$ in magnetic field strength; however, this is still within the maximum uncertainties of magnetic field measurement at 6030 and $6035 \mathrm{MHz}$ as listed in Table 6.

There are many previous magnetic field studies in this source. Fish et al. (2003) used Argon et al. (2000) data from the VLA observations (with compatible velocity resolution of $\sim 0.14 \mathrm{~km} \mathrm{~s}^{-1}$ and the absolute position uncertainties of $0.3 \operatorname{arcsec}$ and 1 arcsec in RA and Dec., respectively) to match possible Zeeman pairs only from the brightest LHCP and RHCP features and estimated the magnetic field value of $-5.3 \mathrm{mG}$ at $1665 \mathrm{MHz}$; this Zeeman pair is associated with our Group 3 at the reference centre as listed in Table 6 . They also provided a wide range of possible magnetic field strengths from -2.2 to $-5.2 \mathrm{mG}$ at $1667 \mathrm{MHz}$ due to ambiguous Zeeman pair matching $\sim 0.1$ arcsec away from the reference centre. Our magnetic field measurements at the reference centre from 
Table 5. OH maser features from both LHCP and RHCP for G351.417+0.645 at $6035 \mathrm{MHz}$. Position offsets are relative to the brightest $6035 \mathrm{LHCP}$ feature.

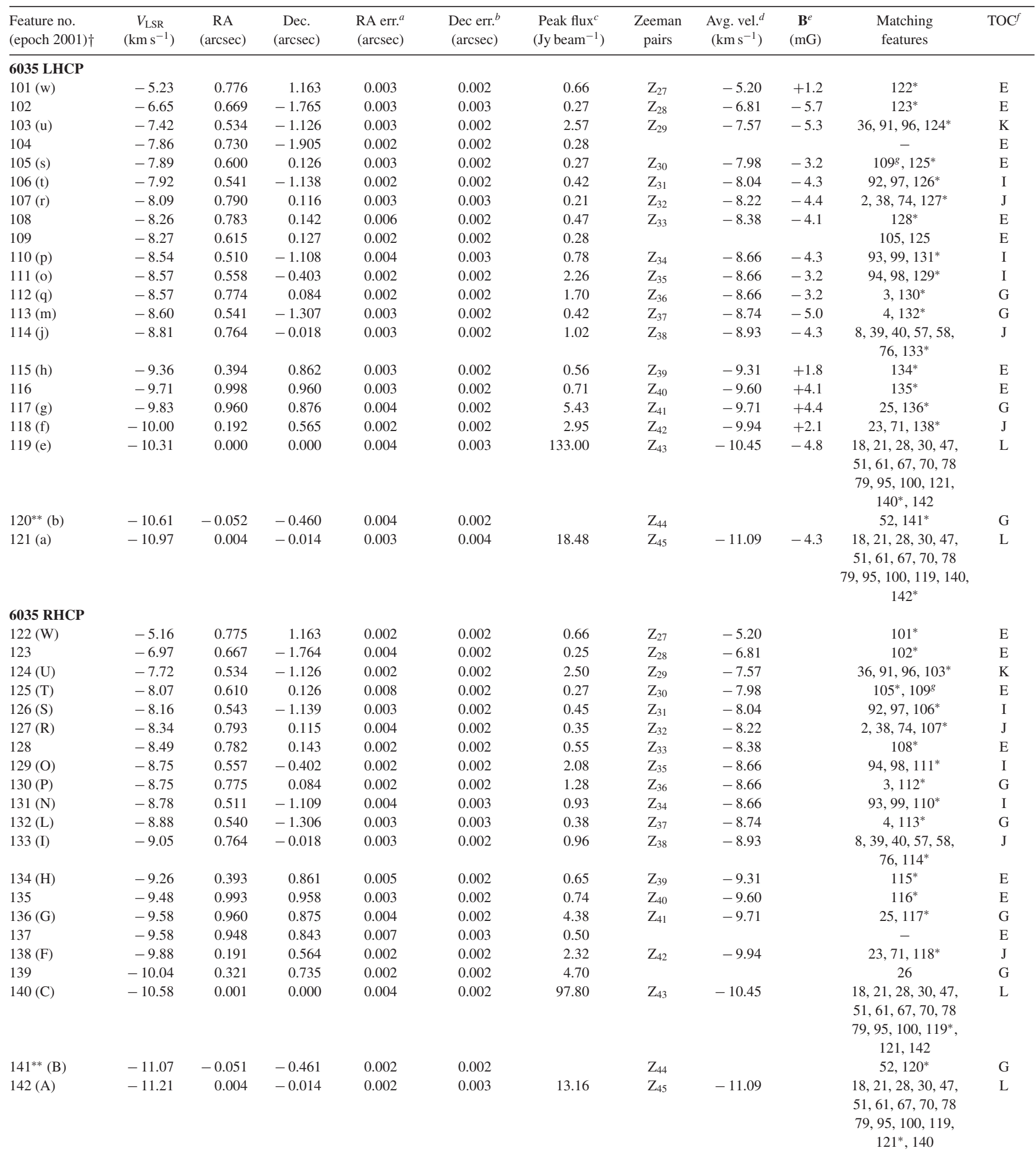

Notes. ${ }^{a, b, d, f, *}$ See Table 2.

$\dagger$ See Table 4.

${ }^{c}$ The peak flux of the brightest component was taken to be the peak flux density of the maser feature. The average rms noise of 6035 -MHz images is $\sim 0.06 \mathrm{Jy}$ with maximum value of $0.7 \mathrm{Jy}$ ( $\sim 0.4$ per cent of the peak flux) in the brightest channel.

${ }^{e} \Delta \mathbf{B}$ is $\pm 1.8 \mathrm{mG}$.

${ }^{8}$ This feature can also be defined as the Zeeman pair of the feature 125 as its position is closer to the feature 125 than that of 105 , but this Zeeman pair provides the magnetic field strength of $+3.5 \mathrm{mG}$ that contradicts to the value of $-2.5 \mathrm{mG}$ from Caswell et al. (2011). However, the Zeeman pair $(105,125)$ or $Z_{30}$ provides the corresponding value of $+3.2 \mathrm{mG}$ which is more sensible in spite of the larger position offset in RA.

** These features are incomplete because they are interfered by side lobes of the brightest features. However, it is still worth listing them for confirming the existence of the Zeeman pair b and B (for LHCP and RHCP, respectively) at $6035 \mathrm{MHz}$ from epoch 2001 (Caswell et al. 2011). 

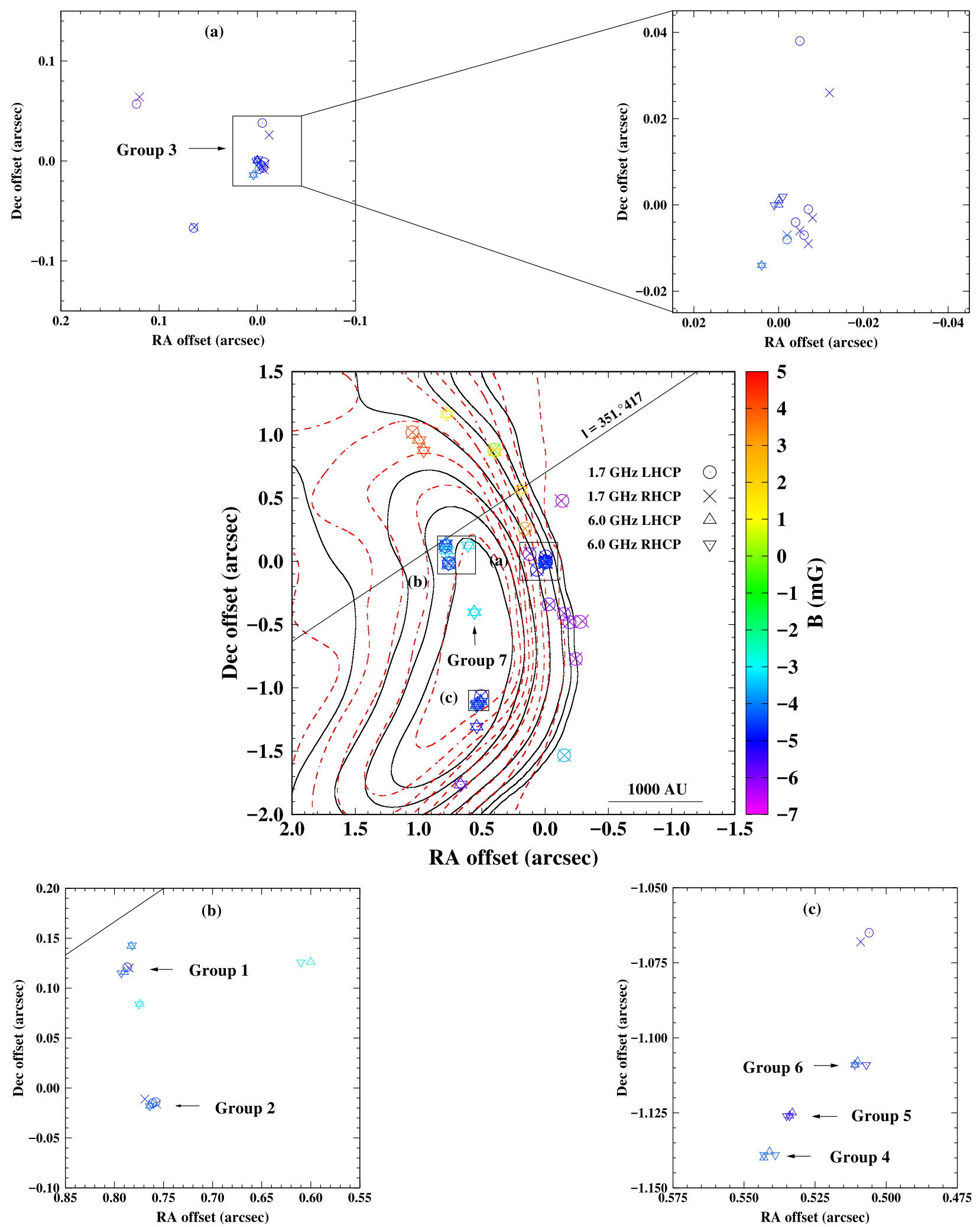

Figure 3. The spatial gradient magnetic fields from all Zeeman pairs of $\mathrm{OH}$ masers at $1.7 \mathrm{GHz}(1665,1667$, and $1720 \mathrm{MHz})$ and $6.0 \mathrm{GHz}(6030 \mathrm{and}$ $6035 \mathrm{MHz}$ ). The colours represent the magnetic field values (the negative and positive signs imply the magnetic field direction directed towards and away from us, respectively). The dash and solid contours are described in Fig. 2. The group numbers show different locations with an association of Zeeman pairs as explained in Table 6. 
Table 6. OH Zeeman pair association (from the same TOC as listed in Tables 2-5) from different transitions at different locations.

\begin{tabular}{|c|c|c|c|c|c|c|c|c|c|c|}
\hline $\begin{array}{l}\text { Group } \\
\text { no. }\end{array}$ & $\begin{array}{c}\text { Transition } \\
(\mathrm{MHz})\end{array}$ & Zeeman pair & $\begin{array}{c}\mathrm{RA}^{g} \\
(\operatorname{arcsec})\end{array}$ & $\begin{array}{c}\operatorname{Dec}^{g} \\
(\operatorname{arcsec})\end{array}$ & $\begin{array}{l}\text { Avg. vel. } \\
\left(\mathrm{km} \mathrm{s}^{-1}\right)\end{array}$ & $\begin{array}{c}\text { B } \\
(\mathrm{mG})\end{array}$ & Ref. & $\Delta \Theta^{h}$ & $\begin{array}{c}\Delta V^{i} \\
\left(\mathrm{~km} \mathrm{~s}^{-1}\right)\end{array}$ & $\begin{array}{l}\Delta \mathbf{B}^{e} \\
(\mathrm{mG})\end{array}$ \\
\hline 1 & $\begin{array}{l}1665 \\
6035 \\
6035\end{array}$ & $\begin{array}{c}\mathrm{Z}_{2} \\
\mathrm{Z}_{32} \\
\text { no. } 18\end{array}$ & $\begin{array}{l}0.786 \\
0.792 \\
0.791\end{array}$ & $\begin{array}{l}0.120 \\
0.116 \\
0.120\end{array}$ & $\begin{array}{l}-8.09 \\
-8.22 \\
-8.16\end{array}$ & $\begin{array}{l}-4.9 \\
-4.4 \\
-4.2\end{array}$ & $\begin{array}{l}{[0]} \\
{[0]} \\
{[1]}\end{array}$ & $\begin{array}{l}0.03 \operatorname{arcsec} \times 0.013 \operatorname{arcsec} \\
0.05 \operatorname{arcsec} \times 0.015 \operatorname{arcsec} \\
0.05 \operatorname{arcsec} \times 0.015 \operatorname{arcsec}\end{array}$ & $\begin{array}{c}0.09 \\
0.1 \\
0.1\end{array}$ & $\begin{array}{l} \pm 0.2 \\
\pm 1.8 \\
\pm 1.8\end{array}$ \\
\hline 2 & $\begin{array}{l}1665 \\
1667 \\
6035 \\
6035 \\
6035\end{array}$ & $\begin{array}{c}\mathrm{Z}_{4} \\
\mathrm{Z}_{12} \\
\mathrm{Z}_{38} \\
\text { no. } 10 \\
\text { UCHII-OH5-1 }\end{array}$ & $\begin{array}{l}0.758 \\
0.765 \\
0.764 \\
0.762 \\
0.766\end{array}$ & $\begin{array}{r}-0.015 \\
-0.013 \\
-0.018 \\
-0.016 \\
0.070\end{array}$ & $\begin{array}{l}-8.83 \\
-8.57 \\
-8.93 \\
-8.90 \\
-8.78\end{array}$ & $\begin{array}{l}-4.5 \\
-4.6 \\
-4.3 \\
-4.3 \\
-4.8\end{array}$ & $\begin{array}{c}{[0]} \\
{[0]} \\
{[0]} \\
{[1]} \\
{[2] ?}\end{array}$ & $\begin{array}{l}0.03 \operatorname{arcsec} \times 0.013 \operatorname{arcsec} \\
0.79 \operatorname{arcsec} \times 0.25 \operatorname{arcsec}\end{array}$ & 0.1 & \pm 1.8 \\
\hline 3 & $\begin{array}{l}1665 \\
1665 \\
1665 \\
1665 \\
1665 \\
1665 \\
1667 \\
1667 \\
1667 \\
6030 \\
6030 \\
6030 \\
6035 \\
6035 \\
6035 \\
6035 \\
6035 \\
6035\end{array}$ & $\begin{array}{c}\mathrm{Z}_{5} \\
\mathrm{Z}_{6} \\
\mathrm{Z}_{8} \\
- \\
- \\
- \\
\mathrm{Z}_{14} \\
\mathrm{Z}_{15} \\
- \\
\mathrm{Z}_{26} \\
\text { no. 24 } \\
\text { UCHII-OH4-1b } \\
\mathrm{Z}_{43} \\
\text { no. 5 } \\
\text { UCHII-OH4-1b } \\
\mathrm{Z}_{45} \\
\text { no. 1 } \\
\text { UCHII-OH4-1a }\end{array}$ & $\begin{array}{r}-0.005 \\
0.026 \\
-0.002 \\
-0.029 \\
-0.003 \\
-0.014 \\
-0.008 \\
-0.007 \\
0.021 \\
-0.001 \\
0.000 \\
0.000 \\
0.001 \\
0.000 \\
0.000 \\
0.004 \\
0.005 \\
0.000\end{array}$ & $\begin{array}{r}-0.005 \\
-0.009 \\
-0.008 \\
0.016 \\
-0.020 \\
-0.009 \\
-0.002 \\
-0.008 \\
-0.034 \\
0.002 \\
0.000 \\
0.010 \\
0.000 \\
0.000 \\
0.000 \\
-0.014 \\
-0.016 \\
0.000\end{array}$ & $\begin{array}{l}-10.29 \\
-10.28 \\
-10.10 \\
-10.55 \\
-10.43 \\
-10.48 \\
-10.58 \\
-10.52 \\
-11.11 \\
-10.45 \\
-10.47 \\
-10.50 \\
-10.45 \\
-10.40 \\
-10.40 \\
-11.00 \\
-11.07 \\
-11.03\end{array}$ & $\begin{array}{l}-5.0 \\
-4.9 \\
-4.4 \\
-5.6 \\
-5.3 \\
-6 \\
-5.1 \\
-5.4 \\
-5 \\
-4.9 \\
-4.9 \\
-5.0 \\
-4.8 \\
-4.8 \\
-4.9 \\
-4.3 \\
-4.6 \\
-4.6\end{array}$ & $\begin{array}{c}{[0]} \\
{[0]^{j}} \\
{[0]} \\
{[3]^{*}} \\
{[4]^{*}} \\
{[5]^{*}} \\
{[0]} \\
{[0]} \\
{[5]^{*}} \\
{[0]} \\
{[1]} \\
{[2]} \\
{[0]} \\
{[1]} \\
{[2]} \\
{[0]} \\
{[1]} \\
{[2]}\end{array}$ & $\begin{array}{c}\text { not reported } \\
1.5 \text { arcsec } \\
0.015 \operatorname{arcsec} \\
\\
0.015 \operatorname{arcsec} \\
0.05 \operatorname{arcsec} \times 0.015 \operatorname{arcsec} \\
0.05 \operatorname{arcsec} \times 0.015 \operatorname{arcsec} \\
0.79 \operatorname{arcsec} \times 0.25 \operatorname{arcsec}\end{array}$ & $\begin{array}{l}0.2 \\
0.1 \\
0.1 \\
0.1\end{array}$ & $\begin{array}{l} \pm 0.6 \\
\pm 1.3 \\
\pm 1.3 \\
\pm 1.3\end{array}$ \\
\hline 4 & $\begin{array}{l}6030 \\
6030 \\
6030 \\
6035 \\
6035 \\
6035\end{array}$ & $\begin{array}{c}\mathrm{Z}_{22} \\
\text { no. } 28 \\
\text { UCHII-OH2-2c } \\
\mathrm{Z}_{29} \\
\text { no. } 21 \\
\text { UCHII-OH2-2c }\end{array}$ & $\begin{array}{l}0.534 \\
0.534 \\
0.535 \\
0.534 \\
0.534 \\
0.523\end{array}$ & $\begin{array}{l}-1.126 \\
-1.123 \\
-1.130 \\
-1.126 \\
-1.123 \\
-1.130\end{array}$ & $\begin{array}{l}-7.57 \\
-7.56 \\
-7.54 \\
-7.57 \\
-7.57 \\
-7.52\end{array}$ & $\begin{array}{l}-5.3 \\
-5.2 \\
-5.4 \\
-5.3 \\
-5.5 \\
-5.3\end{array}$ & $\begin{array}{l}{[0]} \\
{[1]} \\
{[2]} \\
{[0]} \\
{[1]} \\
{[2]}\end{array}$ & & & \\
\hline 5 & $\begin{array}{l}6030 \\
6030 \\
6030 \\
6035 \\
6035 \\
6035\end{array}$ & $\begin{array}{c}\mathrm{Z}_{23} \\
\text { no. } 27 \\
\text { UCHII-OH2-2b } \\
\mathrm{Z}_{31} \\
\text { no. } 20 \\
\text { UCHII-OH2-2b }\end{array}$ & $\begin{array}{l}0.541 \\
0.542 \\
0.535 \\
0.542 \\
0.539 \\
0.523\end{array}$ & $\begin{array}{l}-1.140 \\
-1.135 \\
-1.130 \\
-1.139 \\
-1.131 \\
-1.130\end{array}$ & $\begin{array}{l}-8.06 \\
-8.04 \\
-8.00 \\
-8.04 \\
-8.03 \\
-8.00\end{array}$ & $\begin{array}{l}-4.3 \\
-4.6 \\
-4.7 \\
-4.3 \\
-4.3 \\
-4.4\end{array}$ & $\begin{array}{l}{[0]} \\
{[1]} \\
{[2]} \\
{[0]} \\
{[1]} \\
{[2]}\end{array}$ & & & \\
\hline 6 & $\begin{array}{l}6030 \\
6030 \\
6030 \\
6030 \\
6035 \\
6035 \\
6035 \\
6035\end{array}$ & $\begin{array}{c}\mathrm{Z}_{24} \\
\text { no. } 25 \\
\mathrm{UCHII-OH2-1b} \\
\mathrm{UCHII-OH2-2a} \\
\mathrm{Z}_{34} \\
\text { no. } 11 \\
\text { no. } 16 \\
\mathrm{UCHII-OH2-2a}\end{array}$ & $\begin{array}{l}0.509 \\
0.509 \\
0.511 \\
0.535 \\
0.511 \\
0.507 \\
0.510 \\
0.523\end{array}$ & $\begin{array}{l}-1.109 \\
-1.108 \\
-1.120 \\
-1.130 \\
-1.109 \\
-1.105 \\
-1.105 \\
-1.130\end{array}$ & $\begin{array}{l}-8.64 \\
-8.67 \\
-8.59 \\
-8.59 \\
-8.66 \\
-8.86 \\
-8.63 \\
-8.63\end{array}$ & $\begin{array}{l}-5.1 \\
-4.9 \\
-4.7 \\
-4.7 \\
-4.3 \\
-4.3 \\
-4.3 \\
-3.8\end{array}$ & $\begin{array}{l}{[0]} \\
{[1]} \\
{[2]} \\
{[2]} \\
{[0]} \\
{[1]} \\
{[1]} \\
{[2]}\end{array}$ & & & \\
\hline 7 & $\begin{array}{l}6030 \\
6030 \\
6030 \\
6035\end{array}$ & $\begin{array}{c}\mathrm{Z}_{25} \\
\text { no. } 26 \\
\text { UCHII-OH3-1 } \\
\mathrm{Z}_{35}\end{array}$ & $\begin{array}{l}0.559 \\
0.559 \\
0.560 \\
0.558\end{array}$ & $\begin{array}{l}-0.402 \\
-0.401 \\
-0.400 \\
-0.403\end{array}$ & $\begin{array}{l}-8.63 \\
-8.58 \\
-8.58 \\
-8.66\end{array}$ & $\begin{array}{l}-3.4 \\
-3.7 \\
-3.7 \\
-3.2\end{array}$ & $\begin{array}{l}{[0]} \\
{[1]} \\
{[2]} \\
{[0]}\end{array}$ & & & \\
\hline
\end{tabular}


Table 6 - continued

\begin{tabular}{lccccccccc}
\hline $\begin{array}{l}\text { Group } \\
\text { no. }\end{array}$ & $\begin{array}{c}\text { Transition } \\
(\mathrm{MHz})\end{array}$ & Zeeman pair & $\begin{array}{c}\mathrm{RA}^{g} \\
(\operatorname{arcsec})\end{array}$ & $\begin{array}{c}\text { Dec. }{ }^{g} \\
(\operatorname{arcsec})\end{array}$ & $\begin{array}{c}\text { Avg. vel. } \\
\left(\mathrm{km} \mathrm{s}^{-1}\right)\end{array}$ & $\begin{array}{c}\mathbf{B} \\
(\mathrm{mG})\end{array}$ & Ref. & $\begin{array}{c}\Delta \Theta^{h} \\
\left(V^{i}\right.\end{array} \begin{array}{c}\Delta \mathbf{B}^{e} \\
\left(\mathrm{mG}^{-1}\right)\end{array}$ \\
\hline & 6035 & no. 15 & 0.558 & -0.402 & -8.61 & -3.2 & {$[1]$} & & \\
& 6035 & UCHII-OH3-1 & 0.560 & -0.390 & -8.62 & -3.4 & {$[2]$} \\
\hline
\end{tabular}

Notes. ${ }^{g}$ The average positions between LHCP and RHCP features for each Zeeman pair as listed in Tables 2-5.

${ }^{h}$ Angular resolution.

${ }^{i}$ Velocity resolution.

${ }^{e}$ The maximum uncertainty of $\mathbf{B}$ measurement as explained in the footnote of Tables 2-5.

${ }^{e, h, i}$ The values are mentioned only once for each transition and each work.

${ }^{j}$ This Zeeman pair is not coincident with the other Zeeman pairs from our results, it is included in this group due to its corresponding average velocity and magnetic field strength.

${ }^{k}$ The reported value in their work.

? This is probably the blended Zeeman pair of our $6035-\mathrm{MHz} \mathrm{Z}_{32}$ and $\mathrm{Z}_{12}$ Zeeman pairs in our Groups 1 and 2 , respectively.

*The position is shifted so that their brightest 1665-LHCP feature is the same as our result.

[0] This work (LBA: ATCA, Ho, Cd, Mp, Pa).

[1] Caswell et al. (2011) (LBA: ATCA, Mo, Pa).

[2] Hunter et al. (2018) (VLA).

[3] Gaume \& Mutel (1987) (VLA).

[4] Argon et al. (2000) and Fish et al. (2003) (VLA).

[5] Zheng (1989) (VLBI MK III: VLA, NRAO, HRAS).

the brightest Zeeman pairs at 1665 and $1667 \mathrm{MHz}$ are -5.0 and $-5.1 \mathrm{mG}$, respectively. At $1665 \mathrm{MHz}$, our magnetic field value and Fish et al. (2003)'s agree within each other's measurement errors and we resolve their ambiguous magnetic field value measurement at $1667 \mathrm{MHz}$. In addition, a Zeeman pair at $1720 \mathrm{MHz}$ was also established in Fish et al. (2003)'s work, but they calculated a magnetic field strength of $-3.1 \mathrm{mG}$ by using the Zeeman coefficient of $0.236 \mathrm{~km} \mathrm{~s}^{-1} \mathrm{mG}^{-1}$. However, using the Zeeman coefficient adopted for the current work for this transition (i.e. $0.113 \mathrm{~km} \mathrm{~s}^{-1}$ $\mathrm{mG}^{-1}$ ) leads to a magnetic field strength of $-6.5 \mathrm{mG}$ for this Zeeman pair which is in good agreement with our value of $-6.3 \mathrm{mG}$ from our brightest Zeeman pair. Besides, there are VLA observations of Gaume \& Mutel (1987) (with poorer velocity resolution and absolute position uncertainty of $1.1 \mathrm{~km} \mathrm{~s}^{-1}$ and 0.3 arcsec, respectively) that estimated the magnetic field value to be $-5.6 \mathrm{mG}$ from matched Zeeman pair of the brightest features at $1665 \mathrm{MHz}$ (while there is not any matched Zeeman pair at $1667 \mathrm{MHz}$ ). At $1720 \mathrm{MHz}$, they reported a magnetic field value of $-6.4 \mathrm{mG}$ from the brightest Zeeman pair. This shows that much lower velocity resolution observations from Gaume \& Mutel (1987) still provide corresponding magnetic field values compared to ours and Argon et al. (2000)'s.

There are also previous VLBI observations of Zheng (1989) with high spatial resolution but low velocity resolution $(\sim 0.015$ arcsec and $\sim 0.2 \mathrm{~km} \mathrm{~s}^{-1}$, respectively). They found 4 and 1 matched Zeeman pairs at 1665 and $1667 \mathrm{MHz}$, respectively, and provided magnetic field values ranging from -5 to $-7 \mathrm{mG}$. At 1665 and $1667 \mathrm{MHz}$, they reported magnetic field values of -6 and $-5 \mathrm{mG}$, respectively, near our reference centre as listed in Table 6. Compared with our results at the centre, their magnetic field values are $\sim 1 \mathrm{mG}$ higher at $1665 \mathrm{MHz}$ and correspondent at $1667 \mathrm{MHz}$.

The recent work of Hunter et al. (2018) also measured the spatial gradient magnetic fields in this source using the VLA with comparable velocity resolution of $0.1 \mathrm{~km} \mathrm{~s}^{-1}$ but lower spatial resolution of $0.79 \operatorname{arcsec} \times 0.25$ arcsec. Their results show corresponding magnetic field strengths and demagnetized velocities for all associated Zeeman pairs; some of them are demonstrated in our Table 6 (their brightest $6035-\mathrm{MHz}$ LHCP feature is set as reference centre). Their 6035-MHz Zeeman pair UCHII-OH5-1 is located between our two
6035-MHz Zeeman pairs in Group 1 and Group 2; it is possible that our two Zeeman pairs are blended in UCHII-OH5-1. We can spatially resolve their blended Zeeman pairs reported at the same position at both 6030 - and $6035-\mathrm{MHz}$ transitions by considering the magnetic field strengths and demagnetized velocities. At $6030 \mathrm{MHz}$, their UCHII-OH2-2a, UCHII-OH2-2b, and UCHII-OH2-2c match with our $Z_{24}, Z_{23}$, and $Z_{22}$ in our Groups 6, 5 and 4, respectively. In the same way, at $6035 \mathrm{MHz}$, their UCHII-OH2-2a, UCHII-OH2-2b, and UCHII-OH2-2c are coincident with our $\mathrm{Z}_{34}, \mathrm{Z}_{31}$, and $\mathrm{Z}_{29}$ in our Groups 6, 5 and 4, respectively. In addition, in Group 3 at $6035 \mathrm{MHz}$ their brightest Zeeman pair UCHII-OH4-1a and the weaker coincident Zeeman pair UCHII-OH4-1b also match with our $\mathrm{Z}_{43}$ and $\mathrm{Z}_{45}$ that are also our brightest and weaker coincident Zeeman pairs, respectively.

Magnetic fields in this region were also studied using Parkes single dish observations with compatible velocity resolution of $\sim 0.1$ $\mathrm{km} \mathrm{s}^{-1}$. Caswell (2004) observed 1720-MHz OH maser emission and estimated the magnetic field value to be $-6.4 \mathrm{mG}$ from LHCP and RHCP spectrum splitting that is in good agreement with our value. Caswell (2003) and Caswell \& Vaile (1995) observed $\mathrm{OH}$ maser emission at 6030 and $6035 \mathrm{MHz}$ and derived the magnetic field of $-4.0 \mathrm{mG}$ from LHCP and RHCP spectrum splitting. The lower magnetic field possibly come from the blending of all the Zeeman pairs inside the velocity range of the LHCP and RHCP spectrum peaks. However, we confirm the agreement of the magnetic field from previous single dish observations with our results if considering the maximum measurement uncertainties at $6.0 \mathrm{GHz}$ (i.e. \pm 1.3 and $\pm 1.8 \mathrm{mG}$ at 6030 and $6035 \mathrm{MHz}$, respectively).

These magnetic field comparisons allow us to prove the reliability of the magnetic field measurements from previous lower resolution observations. We confirm that magnetic field values from previous lower resolution measurements are still in good agreement with those from higher resolution measurements. However, higher resolution observation can provide more details of spatial gradient magnetic fields.

Furthermore, comparable LBA observations of Caswell et al. (2011) found 28 Zeeman pairs at both 6030 and $6035 \mathrm{MHz}$ as listed in their table 1 , providing magnetic field strengths ranging from -5.4 to $+6.4 \mathrm{mG}$. However, our magnetic fields at $6.0 \mathrm{GHz}$ range 
from -5.4 to $+4.4 \mathrm{mG}$ as listed in Tables $2-5$; a narrower magnetic field range is due to the vanishing of two Zeeman pairs $\left(Z_{3}\right.$ and $\mathrm{Z}_{4}$ ) from Caswell et al. (2011)'s work with magnetic fields of +6.4 and $+5.2 \mathrm{mG}$. There are the same 21 Zeeman pairs from both 6030 and $6035 \mathrm{MHz}$ from two epochs. The magnetic field variations are within $1 \mathrm{mG}$ (which is less than the maximum uncertainties of magnetic field measurement) for all the same Zeeman pairs and the directions of magnetic field are the same. A very good agreement between magnetic field strengths and directions and demagnetized velocities (within $0.1 \mathrm{~km} \mathrm{~s}^{-1}$ ) estimated at these two epochs as well as the trend of spatial distributions imply quite stable environmental conditions over an 11-yr time.

\subsubsection{Interpretation of the reversal of magnetic field}

The assumptions of reversal of magnetic fields were attributed in Caswell et al. (2011)'s analysis to the variation of magnetic field lines (along Galactic longitude) of clockwise Galactic magnetic field perpendicular to the line of sight. In this work, we propose a possible cause for the alteration of the direction of the magnetic field lines in this region.

Fig. 3 shows the spatial gradient magnetic fields along the northwest boundary of the dust clump MM3 (black contours) and the UCHII region NGC 6334F (red contours) as described in Fig. 2. It can be seen that the positive magnetic field positions (yellow to red features) are distributed in the overlapping region from two internal fragmented dust clumps inside MM3 or NGC 6334F that is also connected to the external clump MM1 above the NGC 6334F as shown in the top image of Fig. 2.

Recent FIR polarimetry observations of Dotson et al. (2010), Zhang et al. (2014) that are summarized in Li et al. (2015) provide the basis for a clearer picture of the observed changes of the magnetic field lines from large to smaller scales. Zhang et al. (2014) provide the polarization image and the magnetic field lines in the sky plane direction in the region NGC 6334I from their $870 \mu \mathrm{m}$ polarimetric observation in their fig. 1(d) showing the magnetic field lines passing through the dust clumps MM1 and MM2 located in the north and north-west of MM3, while, unfortunately, they did not detect MM3 in $870 \mu \mathrm{m}$. Additionally, the $\mathrm{CH}_{3} \mathrm{OH}$ polarization observations of Dodson \& Moriarty (2012) show the corresponding trend of magnetic field lines to those of Zhang et al. (2014)'s near the overlapping region where magnetic field values are positive implying there should be some significant influence from MM1 clump to smaller scale magnetic field lines in the north of MM3 clump (related to maser emission, i.e. $\mathrm{CH}_{3} \mathrm{OH}$ and $\mathrm{OH}$ ). To clarify this, the dust polarimetric observation and maser polarimetric study of both MM1 and MM3 together are necessary.

Based on Li et al. (2015), in the smaller scale, the large-scale magnetic field permeating the region can be compressed and subsequently bent by the fragmented dust clumps, resulting in different directions of magnetic field lines between clumps. As a result, for the two overlapping clumps in MM3 (supported by two peaks of the NGC 6334F contours) magnetic field lines can be distorted in the overlapping region providing the observed reversal of magnetic field direction in our 2D perspective.

This assumption supports the idea of Caswell et al. (2011) that for a predominantly perpendicular magnetic field lines to the line of sight, a variation of $\pm 20^{\circ}$ can provide the opposite magnetic field directions in Zeeman splitting effect.

In a general sense, it is also possible for other multiclump regions, similar to NGC 6334F, to exhibit reversal of magnetic fields. For example, the nearby G351.775-0.536 containing two overlapping

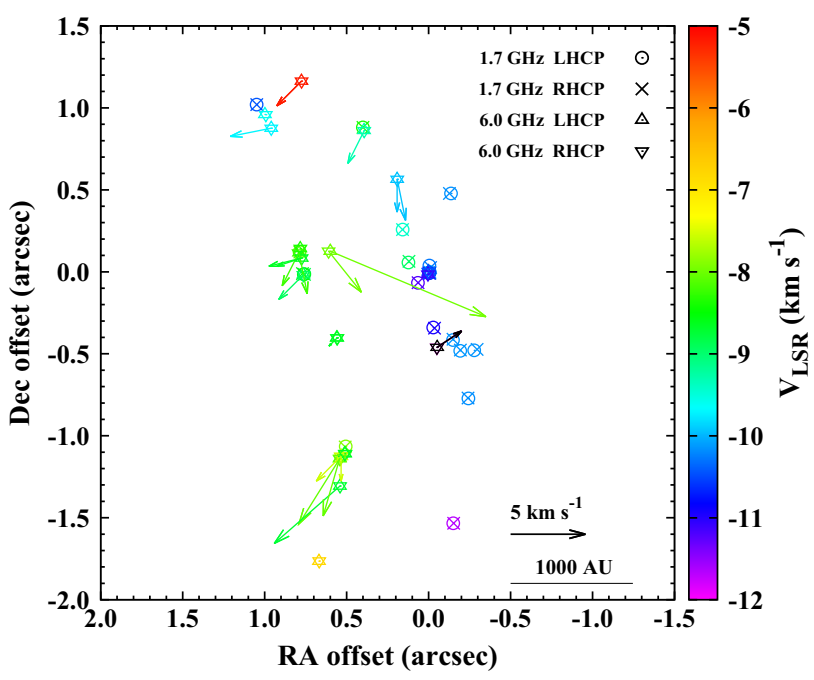

Figure 4. The spatial gradient radial velocities of $\mathrm{OH}$ maser features at 1.7 and $6.0 \mathrm{GHz}$ calculated by averaging the velocities of LHCP and RHCP features of each matched Zeeman pair and the internal proper motions of 6.0-MHz OH maser features relative to the brightest 6035-MHz LHCP. The black features are our incomplete Zeeman pair $Z_{44}$; this is the same Zeeman pair as $Z_{2}$ from Caswell et al. (2011) with demagnetized velocity -10.75 $\mathrm{km} \mathrm{s}^{-1}$ of and magnetic field strength of $-4.8 \mathrm{mG}$.

clumps defined by $8 \mu \mathrm{m}$ contours as shown in fig. 15 of Goedhart, van der Walt \& Gaylard (2002) shows a reversal of magnetic fields from ground-state $\mathrm{OH}$ maser observations as shown in fig. 38 of Fish et al. (2005) with positive magnetic fields in lower Dec. near the overlapping region.

\subsection{Kinematics}

We calculated the demagnetized radial velocity of $\mathrm{OH}$ maser features at 1.7 and $6.0 \mathrm{GHz}$ by averaging the velocities of LHCP and RHCP OH maser features of each Zeeman pairs. Fig. 4 shows the spatial radial velocity gradient from 21 and 24 Zeeman pairs at 1.7 and $6.0 \mathrm{GHz}$, respectively, with velocity range between -11.7 and $-5.2 \mathrm{~km} \mathrm{~s}^{-1}$ as listed in column 9 of Tables $2-5$. This range does not much differ from the systemic velocity of $-10 \mathrm{~km} \mathrm{~s}^{-1}$. There are two $\mathrm{OH}$ maser features at the highest and lowest positions in Dec. showing clearly high red shift with radial velocities of -5.2 and $-6.8 \mathrm{~km} \mathrm{~s}^{-1}$ (i.e. $\mathrm{Z}_{27}$ and $\mathrm{Z}_{28}$, respectively). However, it is still insufficient for interpreting any kinematic property. In comparison with Caswell et al. (2011)'s results, there are no significant changes in radial velocities for the same matched Zeeman pair (the differences are less than a velocity resolution of $\sim 0.1 \mathrm{~km} \mathrm{~s}^{-1}$ ). This infers that all $\mathrm{OH}$ maser features at $6.0 \mathrm{GHz}$ have been moving at apparently constant velocity along the line of sight direction in an 11-yr period.

We also tried to calculate the internal proper motion ${ }^{6}$ by considering the position shifts of the same $42 \mathrm{OH}$ maser features from Caswell et al. (2011)'s (2001 epoch) and our observations (2012 epoch). The actual internal proper motion is defined by using the

\footnotetext{
${ }^{6}$ The internal proper motion is based on the assumption that the brightest LHCP features from 2 epochs (i.e. the features 119 and e of ours and Caswell et al. (2011)'s, respectively) are at the same position at the centre: this represents the actual proper motion relative to the brightest features at the reference centre rather than absolute proper motion.
} 
criteria that the angular displacement of the same feature has to be larger than the position uncertainties. ${ }^{7}$ We assume the position errors are the same for Caswell et al. (2011)'s features. Based on this criteria, $21 \mathrm{OH}$ maser features of all the same 42 features (50 per cent) are defined as actual internal proper motion. The proper motion vectors showing the translational velocities and directions are overlaid on the Fig. 4. The features 105 (or s of Caswell et al. 2011) shows the largest actual proper motion of $\sim 21$ mas corresponding to the maximum transverse velocity of $\sim 11 \mathrm{~km} \mathrm{~s}^{-1}$. It is shown that they generally tend to move downward to both south-east and south-west directions relative to the reference centre.

\subsection{Physical conditions}

Inside HMSFRs, there are many possible pumping mechanisms producing $\mathrm{OH}$ maser emissions: collisions, local line overlap, non-local line overlap and external IR radiation (Pavlakis \& Kylafis 1996a,b). However, various physical conditions inside HMSFRs can allow such mechanisms to happen including the dust temperature $\left(T_{\mathrm{d}}\right)$, the gas kinetic temperature $\left(T_{\mathrm{k}}\right)$, the $\mathrm{H}_{2}$ gas density $\left(n_{\mathrm{H}_{2}}\right)$ and the $\mathrm{OH}$ specific column density $\left(N_{\mathrm{OH}} / \Delta V\right)$ (Cragg, Sobolev \& Godfrey 2002). As a result, $\mathrm{OH}$ maser modellings are very necessary for inferring specific physical conditions for different types of masers or coincidences of masers. In this work, we classify 12 types of coincidence (TOC) of masers from A-L as follows: A (only $1665 \mathrm{MHz}$ ), B (only $1667 \mathrm{MHz}$ ), C (only $1720 \mathrm{MHz}$ ), D (only $6030 \mathrm{MHz}$ ), E (only $6035 \mathrm{MHz}), \mathrm{F}$ (1665 \& $1667 \mathrm{MHz}), \mathrm{G}(1665$ \& $6035 \mathrm{MHz})$, H (1667 \& $6035 \mathrm{MHz}), \mathrm{I}(6030$ \& $6035 \mathrm{MHz}), \mathrm{J}(1665$ \& 1667 \& $6035 \mathrm{MHz}), \mathrm{K}(1665$ \& 6030 \& $6035 \mathrm{MHz})$, and L (1665 \& 1667 \& 6030 \& $6035 \mathrm{MHz})$. We list the TOC associated with each $\mathrm{OH}$ maser feature in column 12 of Tables $2-5$ and summarize the number of $\mathrm{OH}$ maser feature for each TOC as listed in Table 7. From $142 \mathrm{OH}$ maser features detected in this work, most of the $\mathrm{OH}$ maser features or 23.2 per cent ( 33 features) are in TOC A. For the second most abundant TOCs, i.e. TOC J and L have equivalent number of $\mathrm{OH}$ maser features holding totally 24.0 per cent (17 features each). Besides, in decreasing order, TOC E, G, I, B, C, F, and $\mathrm{K}$ share 10.6 per cent (15 features), 9.9 per cent (14 features), 8.5 per cent (12 features), 7.7 per cent (11 features), 7.0 per cent (10 features), 5.6 per cent ( 8 features), and 3.5 per cent ( 5 features) of the total number, respectively. Note that there is not any $\mathrm{OH}$ maser feature in TOC D and $\mathrm{H}$ is found in this work.

To investigate the physical conditions in this region, we compare our results with the $\mathrm{OH}$ maser modellings of Cragg et al. (2002) that was developed from previous models of Sobolev \& Deguchi (1994) to obtain better agreement with the observational data. Note that Cragg et al. (2002)'s OH modellings include only local line overlap; however, their modellings consider the collision rate constants for $48 \mathrm{OH}$ energy levels to obtain more reliable results for the 6.0$\mathrm{GHz}$ transitions compared to previous work of Pavlakis \& Kylafis (2000) that uses only 28 levels. Our criteria for defining physical conditions are mainly based on the TOCs. Fig. 5 shows the distribution of $\mathrm{OH}$ maser features in different colour-coded TOCs. The physical conditions associated to each TOC adopted from Cragg et al. (2002)'s paper are shown in Fig. 6. The area delimitations correspond to the coincidence of the transitions presented in the

\footnotetext{
${ }^{7}$ For internal proper motion, only relative positions to the reference centre are considered, so for the position errors, we use standard deviations $(\sigma)$ for the 6030- and 6035-MHz transitions and the intraband astrometric errors ignoring the effects from thermal noise and interband astrometric errors.
}

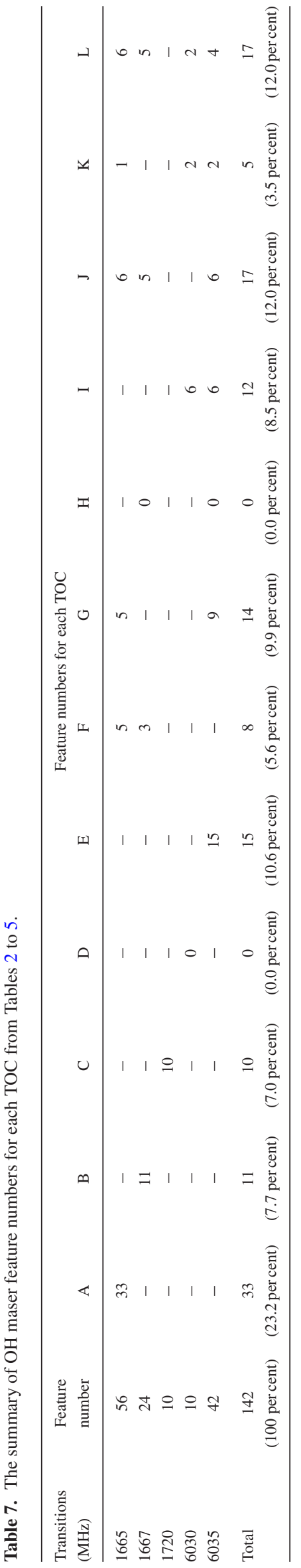



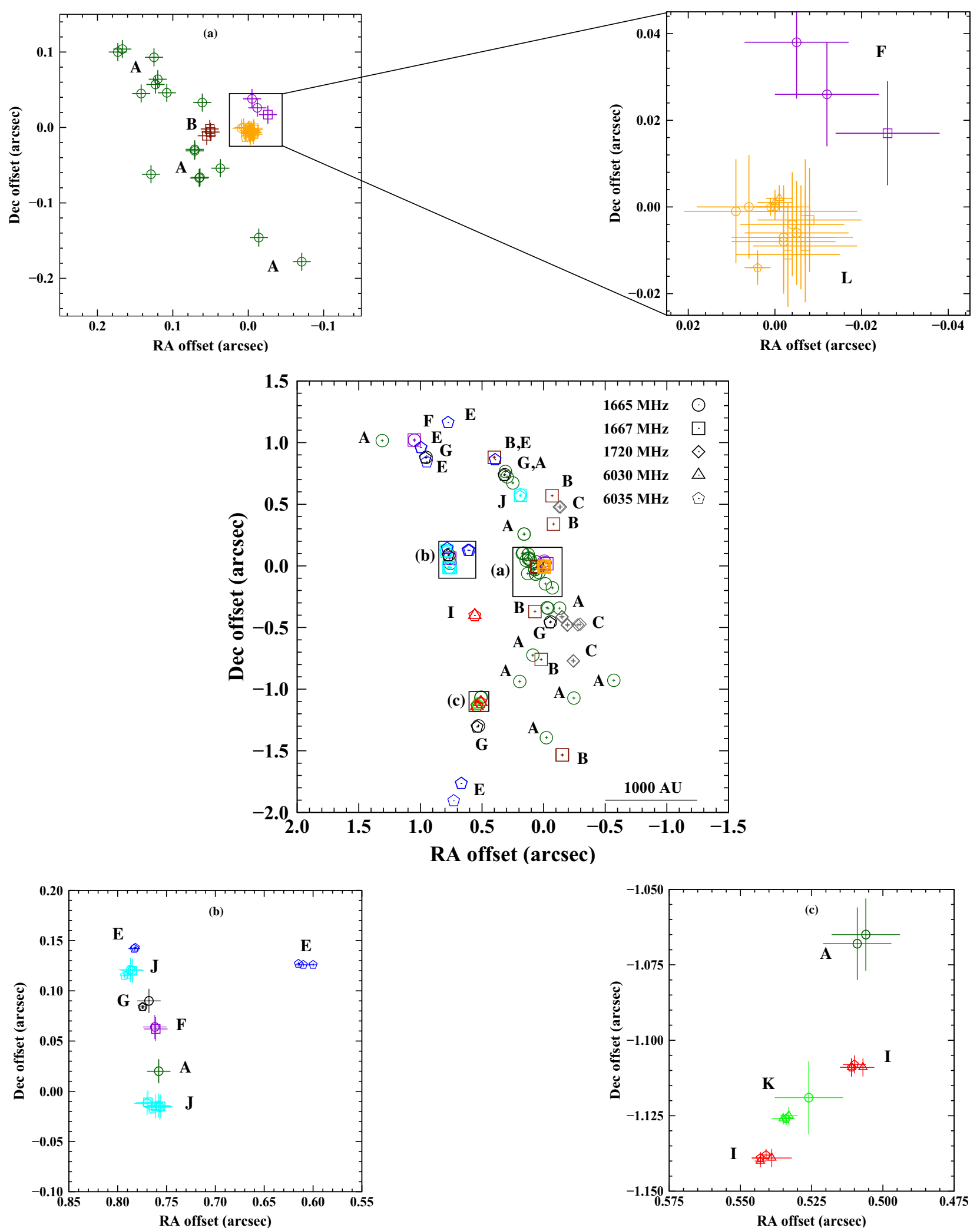

Figure 5. The physical conditions at different positions in these regions based on different TOC as listed in column 12 in Tables $2-5$. The OH maser features are from both LHCP and RHCP. Different colours represent the different TOC as follows: A (dark-green), B (brown), C (grey), E (blue), F (violet), G (black), $\mathrm{I}$ (red), J (cyan), K (yellow-green), L (orange) (there is not any TOC D and H found in this work). 

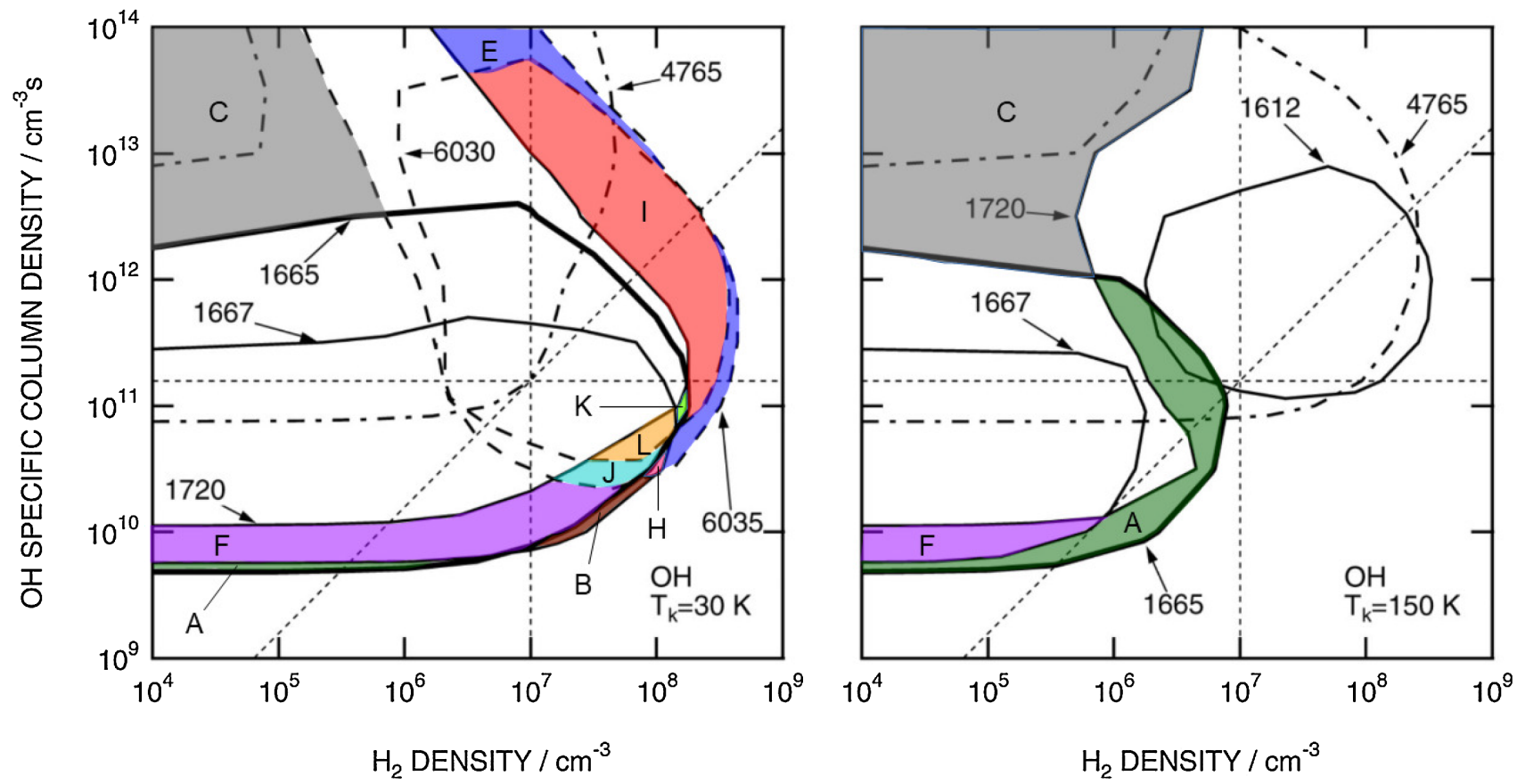

Figure 6. The physical conditions exciting $\mathrm{OH}$ maser emission for different $\mathrm{TOC}$ as a function of $\mathrm{H}_{2}$ density and $\mathrm{OH}$ specific column density at $T_{\mathrm{d}}=175 \mathrm{~K}$ and $T_{\mathrm{k}}=30 \mathrm{~K}$ (left) and $150 \mathrm{~K}$ (right) adapted from the $\mathrm{OH}$ maser modellings of Cragg et al. (2002). Note that we excluded the 4765-MHz line from the interpretation, as it was not observed in this work. The contours trace where the brightness temperature is equal to $10^{4} \mathrm{~K}$. Different colour-coded regions represent the physical conditions corresponding to TOC colours of $\mathrm{OH}$ maser features in Fig. 5.

current work (i.e. hence excluding the 4765-MHz transition appearing in the figure). The contours in Fig. 6 trace the boundary where the brightness temperature is equal to $10^{4} \mathrm{~K}^{8}$ that rapidly increases after those boundaries up to $10^{10}-10^{11} \mathrm{~K}$ and $10^{9}-10^{10}$ $\mathrm{K}$ for $1.7-$ and $6.0-\mathrm{GHz}$ transitions, respectively. Note that Cragg et al. (2002)'s modellings in Fig. 6 do not consider UCHII background radiation. However, their simulations show that brightness temperature decreases with increasing UCHII background radiation as shown in their fig. 7. Only the $1720-\mathrm{MHz} \mathrm{OH}$ maser features are not coincident with $\mathrm{OH}$ maser features from other transitions. From Fig. 6, it is obvious that excluding the 1720-MHz emission region from those of other transitions can more precisely identify the possible condition region for each TOC. Based on this criteria, the physical condition of each TOC can be described and ordered by their significances of interpretation as follows.

TOC L (1665 \& $1667 \& 6030$ \& $6035 \mathrm{MHz} ;$ orange colour): A TOC L cluster is only present near the reference centre as shown in Fig. 5. As illustrated in Fig. 6 where $T_{\mathrm{d}}$ is fixed at $175 \mathrm{~K}$, at low $T_{\mathrm{k}}(30 \mathrm{~K})$ only a narrow region in the parameter space leads to the required conditions for the coincidence of all four transitions: $N_{\mathrm{OH}} / \Delta V$ range of $\sim 10^{10.6}$ to $10^{11} \mathrm{~cm}^{-3} \mathrm{~s}$ and $n_{\mathrm{H}_{2}}$ range of $\sim 10^{7.5}$

\footnotetext{
${ }^{8}$ This corresponds to flux densities of $\sim 0.02$ mJy beam $^{-1}$ for 1665 and $1667 \mathrm{MHz}, \sim 0.4 \mathrm{mJy}^{-1}$ beam ${ }^{-1}$ for $1720 \mathrm{MHz}$ and $\sim 0.7 \mathrm{mJy} \mathrm{beam}^{-1}$ for 6030 and $6035 \mathrm{MHz}$. These are calculated from the relation $T=1.36 \lambda^{2} S / \theta^{2}$ derived from the Rayleigh-Jeans law where $T$ is brightness temperature $(\mathrm{K}), \lambda$ is wavelength $(\mathrm{cm})$, and $\theta$ is HPBW (arcsecond). Note that we used HPBW of 0.03 for 1665 and $1667 \mathrm{MHz}, 0.13$ for $1720 \mathrm{MHz}$, and 0.05 for 6030 and $6035 \mathrm{MHz}$. All of our $\mathrm{OH}$ maser features have higher flux densities than those at the boundaries confirming the possible physical conditions are within the contour boundaries.
}

to $10^{8.2} \mathrm{~cm}^{-3}$. In particular too high $T_{\mathrm{k}}$ leads to the disappearance of the 6030- \& 6035-MHz transitions.

TOC A (only $1665 \mathrm{MHz}$; dark-green colour): A TOC A cluster is observed near the reference centre close to the TOC L cluster but slightly shifted to higher RA as shown in Fig. 5. Besides, some of the isolated $1665-\mathrm{MHz}$ maser features are found along the UCHII region boundary. Fig. 6 shows that at $T_{\mathrm{k}}=30 \mathrm{~K}$ this TOC can be present in very specific $N_{\mathrm{OH}} / \Delta V$ of $\sim 10^{9.7} \mathrm{~cm}^{-3} \mathrm{~s}$ but in a much wider $n_{\mathrm{H}_{2}}$ range of $<10^{4}$ to $10^{6.5} \mathrm{~cm}^{-3}$. However, the region for this TOC increases with increasing $T_{\mathrm{k}}$ providing a much larger region with a $N_{\mathrm{OH}} / \Delta V$ range of $\sim 10^{9.7}$ to $10^{12} \mathrm{~cm}^{-3} \mathrm{~s}$ and a $n_{\mathrm{H}_{2}}$ range of $<10^{4}$ to $10^{6.9} \mathrm{~cm}^{-3}$ at $T_{\mathrm{k}}=150 \mathrm{~K}$. Therefore, this TOC is more likely to be present for high $T_{\mathrm{k}}$. Moreover, fig. 6 of Cragg et al. (2002) suggests that this TOC can be obtained for a $T_{\mathrm{k}}$ ranging from $\sim 90$ to $125 \mathrm{~K}$ at $T_{\mathrm{d}}=175 \mathrm{~K}$ with $N_{\mathrm{OH}} / \Delta V=10^{11.2} \mathrm{~cm}^{-3} \mathrm{~s}$ and $n_{\mathrm{H}_{2}}=10^{7} \mathrm{~cm}^{-3}$. Besides, for the same $N_{\mathrm{OH}} / \Delta V$ and $n_{\mathrm{H}_{2}}$ values, considered at $T_{\mathrm{k}}=150 \mathrm{~K}$, this TOC can also be present when $T_{\mathrm{d}}$ is $>200 \mathrm{~K}$ as shown in fig. 5 of Cragg et al. (2002). Therefore, if this TOC is generated in high $T_{\mathrm{k}}$ and $T_{\mathrm{d}}$ conditions, it is expected to be found in distinctively separated location than TOC L clusters which require low $T_{\mathrm{k}}$.

TOC E (only $6035 \mathrm{MHz}$; blue colour): The isolated 6035$\mathrm{MHz}$ maser features are distributed in a strip RA offset $=[0.3 \operatorname{arcsec} ; 1.0 \operatorname{arcsec}]$ and Dec. offset $=[-2.0 \operatorname{arcsec} ; 1.5 \operatorname{arcsec}]$ as shown in Fig. 5. As illustrated in Fig. 6, there are two possible separate regions in the parameter space for this TOC at low $T_{\mathrm{k}}$ (i.e. $T_{\mathrm{k}}=30 \mathrm{~K}$ ). The first region requires $n_{\mathrm{H}_{2}}$ to be in the range of $\sim 10^{8.1}$ to $10^{8.7} \mathrm{~cm}^{-3}$ and $N_{\mathrm{OH}} / \Delta V$ to be in the range of $\sim 10^{10.5}$ to $10^{12.4}$ $\mathrm{cm}^{-3} \mathrm{~s}$. The second region occurs at much higher $N_{\mathrm{OH}} / \Delta V$ ranging from $\sim 10^{12.9}$ to $>10^{14} \mathrm{~cm}^{-3} \mathrm{~s}$ and wider $n_{\mathrm{H}_{2}}$ range of $<10^{6.2}$ to $10^{8.1} \mathrm{~cm}^{-3}$. 
TOC I (6030 \& $6035 \mathrm{MHz}$; red colour): This TOC is present in a wide range of both $N_{\mathrm{OH}} / \Delta V$ and $n_{\mathrm{H}_{2}}$ conditions at $T_{\mathrm{k}}=30 \mathrm{~K}$ : $\sim 10^{10.9}$ to $10^{13.8} \mathrm{~cm}^{-3} \mathrm{~s}$ and $\sim 10^{6.5}$ to $10^{8.6} \mathrm{~cm}^{-3}$, respectively, and absent at particularly high $T_{\mathrm{k}}$ as shown Fig. 6 . There is a very good agreement between our results and Cragg et al. (2002)'s models: all the $6030-\mathrm{MHz} \mathrm{OH}$ maser features are coincident with a $6035-\mathrm{MHz}$ feature. However, we notice that only 10 in 42 of the $6035-\mathrm{MHz}$ features are coincident with a $6030-\mathrm{MHz}$ feature, which is quite unexpected due to the very large region in the parameter space for this TOC.

TOC K (1665 \& 6030 \& 6035 MHz; yellow-green colour): TOC $\mathrm{K}$ is only present at position $\sim 0.530 \mathrm{arcsec}$ and $-1.125 \operatorname{arcsec}$ in RA and Dec. offsets as shown in Fig. 5(c). Its scarcity is in agreement with the very small region it occupies in the parameter space (at the far bottom of the TOC-I region) with $N_{\mathrm{OH}} / \Delta V$ and $n_{\mathrm{H}_{2}}$ of $\sim 10^{11}$ $\mathrm{cm}^{-3} \mathrm{~s}$ and $10^{8.2} \mathrm{~cm}^{-3}$, respectively, at $T_{\mathrm{k}}=30 \mathrm{~K}$ as illustrated in Fig. 6. A slight increase in $n_{\mathrm{H}_{2}}$ density can alter the TOC from $\mathrm{K}$ to I and the other way around which we assume to be the reason why we found TOC-I clusters close to the TOC-K one (cf. Fig. 5c).

TOC J (1665 \& $1667 \& 6035 \mathrm{MHz}$; cyan colour): TOC J, compared to TOC L at $T_{\mathrm{k}}=30 \mathrm{~K}$, requires lower $N_{\mathrm{OH}} / \Delta V$ in the range of $\sim 10^{10.4}-10^{10.6} \mathrm{~cm}^{-3} \mathrm{~s}$ with nearly the same $n_{\mathrm{H}_{2}}$ range of $\sim 10^{7.2}-10^{8.1} \mathrm{~cm}^{-3}$ as shown in Fig. 6. This TOC is also absent at particularly high $T_{\mathrm{k}}$ not favourable for the $6035 \mathrm{MHz}$ line. From Fig. 5, we found three separate locations where this TOC occurs. One of them is located near the TOC E and G clusters that possibly comes from slightly lower $N_{\mathrm{OH}} / \Delta V$ and $n_{\mathrm{H}_{2}}$ comparing to TOC E as shown in Fig. 6, while the other two clusters are close to a TOC A feature that are from obviously different physical conditions.

TOC B (only $1667 \mathrm{MHz}$; brown colour): TOC B features are found in a very narrow range of RA offset but a much wider range of Dec. offset along the H II region boundary ranging from -1.5 to 0.75 arcsec as shown in Fig. 5. In comparison with TOC J at $T_{\mathrm{k}}$ $=30 \mathrm{~K}$, this TOC requires slightly lower $N_{\mathrm{OH}} / \Delta V$ in the range of $\sim 10^{9.9}$ to $10^{10.5} \mathrm{~cm}^{-3} \mathrm{~s}$ but comparable $n_{\mathrm{H}_{2}}$ in the range of $\sim 10^{7.0}$ to $10^{7.9} \mathrm{~cm}^{-3}$ as illustrated in Fig. 6. This TOC is also absent in higher $T_{\mathrm{k}}$.

TOC F (1665 \& $1667 \mathrm{MHz}$; purple colour): TOC F clusters are observed in three separate locations as shown in Fig. 5. As illustrated in Fig. 6, at $T_{\mathrm{k}}=30 \mathrm{~K}$, this TOC is present in a large possible $n_{\mathrm{H}_{2}}$ range of $<10^{4}$ to $10^{7.8} \mathrm{~cm}^{-3}$ but a much narrower $N_{\mathrm{OH}} / \Delta V$ range of $\sim 10^{9.8}$ to $10^{10.1} \mathrm{~cm}^{-3} \mathrm{~s}$. This TOC can also be observed at high $T_{\mathrm{k}}$ with a comparable $N_{\mathrm{OH}} / \Delta V$ range but decreased possible maximum $n_{\mathrm{H}_{2}}$ of $10^{5.9} \mathrm{~cm}^{-3}$ at $T_{\mathrm{k}}=150 \mathrm{~K}$. In two locations, this TOC is observed close to TOC E and TOC L clusters, though TOC F requires lower $N_{\mathrm{OH}} / \Delta V$ and $n_{\mathrm{H}_{2}}$ at low $T_{\mathrm{k}}$. In the third location, this TOC is close to both a TOC A feature and a TOC G cluster; if we ignore TOC G here, it appears that TOC F can be present in a wide possible $T_{\mathrm{k}}$ range similar to that of TOC A.

TOC G (1665 \& $6035 \mathrm{MHz}$; black colour): We found a TOC $\mathrm{G}$ cluster in five different locations as shown Fig. 5. However, there is no possible region for this TOC in the parameter space of Cragg et al. (2002)'s OH models. As a result, $\mathrm{OH}$ models that can specifically describe the physical conditions for this cluster are needed. We noticed that these TOC G clusters are observed close to several TOCs including TOC A, B, C, E, F, and J. Therefore, we assume similar conditions to those needed for these TOCs to occur are also favourable for TOC G.

TOC H (1667 \& $6035 \mathrm{MHz}$; pink colour): We do not detect any TOC H; the lack of this TOC is in agreement with the very small region in the parameter space, requiring very specific $N_{\mathrm{OH}} / \Delta V$ and $n_{\mathrm{H}_{2}}$ of $10^{10.5} \mathrm{~cm}^{-3} \mathrm{~s}$ and $10^{8} \mathrm{~cm}^{-3}$, respectively, as shown in Fig. 6.
TOC C (1720 MHz; grey colour): TOC C is observed in westernmost locations near the UCHII region boundary. This TOC requires high $N_{\mathrm{OH}} / \Delta V$ ranging from $\sim 10^{12.3}$ to $>10^{14} \mathrm{~cm}^{-3} \mathrm{~s}$ but low $n_{\mathrm{H}_{2}}$ ranging from $<10^{4}$ to $10^{5.9} \mathrm{~cm}^{-3}$ at $T_{\mathrm{k}}=30$ as shown in Fig. 6. Moreover, the region in the parameter space, favourable for this transition to occur in isolation, increase with increasing $T_{\mathrm{k}}$ with possible range of $N_{\mathrm{OH}} / \Delta V=\left[10^{12} ;>10^{14}\right] \mathrm{cm}^{-3} \mathrm{~s}$ and $n_{\mathrm{H}_{2}}$ $=\left[<10^{4} ; 10^{6.7}\right] \mathrm{cm}^{-3}$ at $T_{\mathrm{k}}=150 \mathrm{~K}$. However, in Fig. 6, Cragg et al. (2002)'s OH models seem to provide overprediction of 1720$\mathrm{MHz} \mathrm{OH}$ maser emission suggesting a large range of conditions favouring coincidence with the other transitions. This contradicts our results since we do not find any $1720-\mathrm{MHz} \mathrm{OH}$ maser features in coincidence with another $\mathrm{OH}$ transition.

4.5.1 An example from previous study related to the association of 1.7-/6.0- $\mathrm{GHz} \mathrm{OH}$ and 6.7- $\mathrm{GHz} \mathrm{CH}_{3} \mathrm{OH}$ lines in the source $\mathrm{W} 3(\mathrm{OH})$

The copropagation between different $\mathrm{OH}$ maser transitions (1665-, 1667-, 1720-, 6030-, and 6035-MHz lines) and between $\mathrm{OH}$ and $\mathrm{CH}_{3} \mathrm{OH}$ (6668-MHz line) masers was also previously reported in Etoka et al. (2005)'s paper for the HMSFR W3(OH) as summarized in their fig. 3 .

The strongest association is from 6.0 - and $1.7-\mathrm{GHz}$ transitions especially between 6035 and $1665 \mathrm{MHz}$. Our results also show the same trend as shown in our Table 7 . The other interesting similarities are that all $6030-\mathrm{MHz}$ features are coincident with $6035-\mathrm{MHz}$ features and no $1720-\mathrm{MHz}$ masers are associated with $6.0-\mathrm{GHz}$ and the other ground-state transitions. In their work, weak association is found between $6.7-\mathrm{GHz} \mathrm{CH}_{3} \mathrm{OH}$ masers and $1.7 / 6.0-\mathrm{GHz} \mathrm{OH}$ masers.

Additionally, Etoka et al. also simulated maser gains (negative optical depths) of several $\mathrm{OH}$ maser lines including $1.7-$ and $6.0-\mathrm{GHz}$ transitions as a function of $\mathrm{OH}$ abundance and dust temperature using the accelerated lambda iteration method (Scharmer \& Carlsson 1985). All 1665-, 1667-, 1720-, 6030-, and 6035-MHz transitions show similar gain surface; among them, $1720 \mathrm{MHz}$ is of the lowest gain. As a result, based on their model, the obvious separation between $1720-\mathrm{MHz}$ and the other transitions is reasonable.

\section{CONCLUSIONS}

With the high spatial and velocity resolutions afforded by new LBA observations, we provide well characterized spatial gradient magnetic fields and radial velocities at both ground- and excited-state $\mathrm{OH}$ transitions for the first time. In comparison with previous lower spatial and velocity resolution observations, we verify the reliability of the measured values of magnetic field strength, confirming that our measured magnetic fields are within the uncertainties of previous measurements. Moreover, we confirm the reversal of magnetic fields in this regions with evidence from both 1665- and 6035$\mathrm{MHz} \mathrm{OH}$ Zeeman pairs. This reversal of magnetic fields can be described by the cloud fragmentation inducing a twist of the dominant negative magnetic fields that turn to positive in the overlapping region.

Additionally, we found similar spatial distributions of $\mathrm{OH}$ maser features at both 1.7 and $6.0 \mathrm{GHz}$ along the arc-shaped north-western boundary of UCHII region NGC 6334F.

We compared two epochs (2001 and 2012) of LBA high spatial and velocity resolution observations at $6.0 \mathrm{GHz}$. We found that in 
the 11-yr time span, the spatial gradient magnetic field and radial velocities had no significant changes (within the velocity resolutions and magnetic field measurement uncertainties). Moreover, we estimated the internal proper motions over the 11-yr interval and found that 50 per cent of all the same $\mathrm{OH}$ maser features between those two epochs tended to move downward relative to the reference feature, while the other half were transversely stationary. This suggests that the environmental conditions in this region have been very stable during this period.

We also inferred the physical conditions in this region based on the types of coincidence of $\mathrm{OH}$ maser features from different transitions by comparing with models of $\mathrm{OH}$ maser emission mechanisms from previous work. We provide possible ranges of $\mathrm{OH}$ specific column density, $\mathrm{H}_{2}$ density at particular gas and dust temperatures in different locations of this source.

\section{ACKNOWLEDGEMENTS}

TC thanks the Science Achievement Scholarship of Thailand (SAST) and the International Centre for Radio Astronomy Research (ICRAR) for the financial support. KA acknowledges the financial support obtained from the National Astronomical Research Institute of Thailand (NARIT) for fiscal year 2016-2017. We thank many of the staffs at the CSIRO's Australia Telescope National Facility (ATNF) associated with the LBA project V452. We would also like to thank (1) Dr. Hiroshi Imai from Department of Physics and Astronomy, Graduate School of Science and Engineering, Kagoshima University for useful suggestion in analysis of $\mathrm{OH}$ internal proper motion, (2) Dr. Malcolm Gray and Dr. Sandra Etoka from Jodrell Bank Centre for Astrophysics, School of Physics and Astronomy, the University of Manchester, UK, for their beneficial ideas and suggestion on $\mathrm{OH}$ maser modelling works, (3) Dr. Crystal L. Brogan and Dr. Todd R. Hunter from National Radio Astronomy Observatory (NRAO), USA, for giving permission to use their $5-\mathrm{cm}$ and 3-mm continuum images of the UCHII region NGC 6334F.

\section{REFERENCES}

Argon A. L., Reid M. J., Menten K. M., 2000, ApJS, 129, 159

Argon A. L., Reid M. J., Menten K. M., 2003, ApJ, 593, 925

Asaki Y. et al., 2007, PASJ, 59, 397

Bartkiewicz A., van Langevelde H. J., 2012, in Booth R. S., Vlemmings W. H. T., Humphreys E. M. L., eds, Proc. IAU Symp. 287, Cosmic Masers - from $\mathrm{OH}$ to H0. Cambridge Univ. Press, Cambridge, p. 117

Beuther H., Sridharan T. K., Schilke P., Wyrowski F., Menten K. M., 2000, in Favata F., Kaas A., Wilson A., eds, ESA Special Publ. Vol. 445, Star Formation from the Small to the Large Scale. p. 331, ESA, Noordwijk

Breen S. L., Ellingsen S. P., 2012, in Booth R. S., Vlemmings W. H. T., Humphreys E. M. L., eds, IAU Symp. Vol. 287, Cosmic Masers - from $\mathrm{OH}$ to H0. Cambridge Univ. Press, Cambridge, p. 156

Brogan C. L., Hunter T. R., Cyganowski C. J., Chandler C. J., Friesen R., Indebetouw R., 2016, ApJ, 832, 187

Caswell J. L., 1997, MNRAS, 289, 203

Caswell J. L., 2003, MNRAS, 341, 551

Caswell J. L., 2004, MNRAS, 349, 99

Caswell J. L., Vaile R. A., 1995, MNRAS, 273, 328

Caswell J. L., Kramer B. H., Reynolds J. E., 2011, MNRAS, 414, 1914

Caswell J. L., Green J. A., Phillips C. J., 2013, MNRAS, 431, 1180

Cragg D. M., Sobolev A. M., Godfrey P. D., 2002, MNRAS, 331, 521
Davies R. D., 1974, in Kerr F. J., Simonson S. C., eds, Proc. IAU Symp. 60, Galactic Radio Astronomy. Springer, Dordrecht, p. 275

De Buizer J. M., Pina R. K., Telesco C. M., 2000, in American Astronomical Society Meeting Abstracts. AAS, Washington, DC, p. 1469

De Buizer J. M., Radomski J. T., Piña R. K., Telesco C. M., 2002, ApJ, 580, 305

Dodson R., Moriarty C. D., 2012, MNRAS, 421, 2395

Dotson J. L., Vaillancourt J. E., Kirby L., Dowell C. D., Hildebrand R. H., Davidson J. A., 2010, ApJS, 186, 406

Ellingsen S. P., Voronkov M. A., Cragg D. M., Sobolev A. M., Breen S L., Godfrey P. D., 2007, in Chapman J. M., Baan W. A., eds, Proc. IAU Symp. 242, Astrophysical Masers and Their Environments. Cambridge Univ. Press, Cambridge, p. 213

Etoka S., Cohen R. J., Gray M. D., 2005, MNRAS, 360, 1162

Fish V. L., 2007, in Chapman J. M., Baan W. A., eds, Proc. IAU Symp. 242, Astrophysical Masers and Their Environments. Cambridge Univ. Press, Cambridge, p. 71

Fish V. L., Reid M. J., 2007, ApJ, 656, 952

Fish V. L., Reid M. J., Argon A. L., Menten K. M., 2003, ApJ, 596 , 328

Fish V. L., , Reid M. J., Argon A. L., Zheng X.-W., 2005, ApJS, 160 220

Forster J. R., Caswell J. L., 1989, A\&A, 213, 339

Gaume R. A., Mutel R. L., 1987, ApJS, 65, 193

Goedhart S., van der Walt D. J., Gaylard M. J., 2002, MNRAS, 335, 125

Green J. A., McClure-Griffiths N. M., Caswell J. L., Robishaw T., HarveySmith L., 2012, MNRAS, 425, 2530

Greisen E. W., 2003, Inf. Handl. Astron.- Historical Vistas, 285, 109

Harvey P. M., Gatley I., 1983, ApJ, 269, 613

Hunter T. R., Brogan C. L., Megeath S. T., Menten K. M., Beuther H., Thorwirth S., 2006, ApJ, 649, 888

Hunter T. R. et al., 2018, ApJ, 854, 170

Hutawarakorn B., Cohen R. J., 2005, MNRAS, 357, 338

Kent S. R., Mutel R. L., 1982, ApJ, 263, 145

Kraemer K. E., Jackson J. M., 1999, ApJS, 124, 439

Li H.-B. et al., 2015, Nature, 520, 518

Lo K. Y., Walker R. C., Burke B. F., Moran J. M., Johnston K. J., Ewing M. S., 1975, ApJ, 202, 650

Marseille M. G., van der Tak F. F. S., Herpin F., Jacq T., 2010, A\&A, 522, A40

McBreen B., Fazio G. G., Stier M., Wright E. L., 1979, ApJ, 232, L183

Migenes V. et al., 1999, ApJS, 123, 487

Pavlakis K. G., Kylafis N. D., 1996a, ApJ, 467, 300

Pavlakis K. G., Kylafis N. D., 1996b, ApJ, 467, 309

Pavlakis K. G., Kylafis N. D., 2000, ApJ, 534, 770

Persi P., Roth M., Tapia M., Marenzi A. R., Felli M., Testi L., Ferrari-Toniolo M., 1996, A\&A, 307, 591

Persi P., Tapia M., Felli M., Lagage P. O., Ferrari-Toniolo M., 1998, A\&A, 336,1024

Reid M. J. et al., 2014, ApJ, 783, 130

Rodriguez L. F., Canto J., Moran J. M., 1982, ApJ, 255, 103

Sakai T., Sakai N., Hirota T., Yamamoto S., 2010, ApJ, 714, 1658

Scharmer G. B., Carlsson M., 1985, J. Comput. Phys., 59, 56

Schönrich R., Binney J., Dehnen W., 2010, MNRAS, 403, 1829

Shepherd M. C., 1997, in Hunt G., Payne H., eds, ASP Conf. Ser. Vol. 125, Astronomical Data Analysis Software and Systems VI. Astron. Soc. Pac., San Francisco, p. 77

Slysh V. I., Migenes V., Val'tts I. E., Lyubchenko S. Y., Horiuchi S., Altunin V. I., Fomalont E. B., Inoue M., 2002, ApJ, 564, 317

Sobolev A. M., Deguchi S., 1994, A\&A, 291, 569

Trinidad M. A. et al., 2013, MNRAS, 430, 1309

Walsh A. J., Burton M. G., Hyland A. R., Robinson G., 1998, MNRAS, 301, 640

Zhang Q. et al., 2014, ApJ, 792, 116

Zheng X.-W., 1989, Chin. J. Space Sci., 9, 336 


\section{APPENDIX: ASTROMETRIC ERROR ANALYSIS}

This section concerns the estimate of astrometric errors that are used through this paper arising from different contributions and analysis. These are relevant for the studies based on comparisons between the morphology of the emission from a given transition at multiple epochs, between transitions within the same frequency band, or intraband, (i.e. 1665, 1667, and $1720 \mathrm{MHz} ; 6030$ and $6035 \mathrm{MHz}$ ), and between bands (i.e. 1.7 and $6.0 \mathrm{GHz}$ ), or interband. When more than one error contribution is relevant for the comparison, the errors are added in quadrature.

\section{A1 Thermal-noise position errors in an image}

The relative position errors for emission in the images from a same transition can be calculated using the formula for the thermal noise contribution, as $0.5 \times \mathrm{FWHM} / \mathrm{SNR}$. The estimated astrometric errors for the brightest features are $\sim 1.6$ and $\sim 1.0$ mas, in the images at 1.7 and $6.0 \mathrm{GHz}$, respectively, using typical values for the beamwidths (38 and 15 mas, at 1.7 and $6.0 \mathrm{GHz}$, respectively). Weaker features will have larger errors.

\section{A2 Interband astrometric errors}

We have used phase referencing techniques, with respect to a reference quasar, for the analysis of target emission at both 1665 and $6035 \mathrm{MHz}$ transitions. This allows to astrometrically register the target emission at both transitions. Note that this, combined with the intraband astrometry, results in a bona fide astrometric registration of the five maser transitions observed. Here, we estimate the propagation of astrometric errors in the phase referencing with respect to $1729-373$, which is $\sim 3^{\circ}$ away from the target source $\mathrm{G} 351.417+0.645$.
We use the formulae in (Asaki et al. 2007) to estimate the error budget for our observations, using nominal values for the errors in the total electron content. The dominant contribution to the phase errors arises from the static component of the ionosphere, and is larger at lower frequencies, that is, $108^{\circ}$ and $30^{\circ}$, at 1.7 and $6.0 \mathrm{GHz}$, respectively, using nominal values for the error in the total electron content. These propagate into astrometric errors equal to 11.4 and 1.3 mas, at 1665 and $6035 \mathrm{MHz}$, respectively, using beamwidths 38 and 15 mas.

\section{A3 Intraband astrometric errors}

We have used phase referencing techniques to astrometrically register the emission from the multiple transitions within each of the two bands, 1.7 and $6.0 \mathrm{GHz}$ with respect to 1665 and $6035 \mathrm{MHz}$, respectively. We calculate the propagation of astrometric errors in this case attending to the formula:

Intraband astrometric error $=\left(\frac{\Delta v}{v}\right) \times \Delta s$,

where $\Delta s$ is the errors in the source position used at the correlator, which we measured from the phase referenced maps with respect to the external reference source to be $\sim 400$ mas. The largest error is for the registration between $1665 \mathrm{MHz} \rightarrow 1720 \mathrm{MHz}$, which is 13.2 mas. The estimated errors for $1665 \mathrm{MHz} \rightarrow 1667 \mathrm{MHz}$ and $6035 \mathrm{MHz} \rightarrow 6030 \mathrm{MHz}$ are 0.5 and 0.3 mas, respectively.

This paper has been typeset from a $\mathrm{T}_{\mathrm{E}} \mathrm{X} / \mathrm{L} \mathrm{A} \mathrm{E} \mathrm{X}$ file prepared by the author. 